\title{
The Askey scheme as a four-manifold with corners
}

\author{
Tom H. Koornwinder
}

Received: 6 September 2009 / Accepted: 6 October 2009 / Published online: 28 October 2009

(C) The Author(s) 2009. This article is published with open access at Springerlink.com

\begin{abstract}
Racah and Wilson polynomials with dilated and translated argument are reparametrized such that the polynomials are continuous in the parameters as long as these are nonnegative, and such that restriction of one or more of the new parameters to zero yields orthogonal polynomials lower in the Askey scheme. Geometrically this will be described as a manifold with corners.
\end{abstract}

Keywords Askey scheme $\cdot$ Hypergeometric orthogonal polynomials · Limit relations $\cdot$ Three-term recurrence relations $\cdot$ Manifold with corners

Mathematics Subject Classification (2000) Primary 33C45

\section{Introduction}

A graphical scheme describing the families of hypergeometric orthogonal polynomials and the limit relations between them was published by Askey and Wilson in 1985 as an Appendix to their famous memoir [1] that introduced the Askey-Wilson polynomials. Immediately afterwards, the scheme was slightly extended for the continuous Hahn polynomials, see [2, 4], and it remained stable since then. The scheme was named after Askey by J. Labelle [18] (tableau d'Askey or Askey scheme), who also made a big wall poster displaying the scheme. As often told by Askey, he got the idea for this scheme already at an Oberwolfach meeting in 1977 on "Combinatorics and Special Functions". There Michael Hoare, in connection with his lecture, distributed copies of a sheet (an extension of [7, p. 285, Fig. 2]) which contained

T.H. Koornwinder $(\bowtie)$

Korteweg-de Vries Institute, University of Amsterdam, P.O. Box 94248, 1090 GE Amsterdam, The Netherlands

e-mail: T.H.Koornwinder@uva.nl 
in graphical way a part of the present Askey scheme, and which was received very enthusiastically by the audience.

Since its introduction, the Askey scheme (given here in Fig. 1) has been very influential both by its compact way of presenting the various families of hypergeometric orthogonal polynomials and by the structuring effect of the arrows which indicate the limit relations. Furthermore, the report [15] by Koekoek and Swarttouw has been very helpful in giving the most important formulas for each family and each limit relation in the scheme (and in the $q$-Askey scheme as well).

Personally, I was in particular intrigued by the arrows. In 1993, I wrote a report [17] in which, for a part of the Askey scheme, limit relations are described together in a uniform way. In different notation, these results are given in the present paper in Sect. 7.1 and the corresponding part of the Askey scheme is in the first graph of Fig. 2. The idea here is to reparametrize the four-parameter family of Racah polynomials, together with a parameter dependent translation and dilation of the argument, such that the resulting orthogonal polynomials become continuous up to the boundary in their dependence on the four nonnegative parameters. Restriction to some part of the boundary yields families lying below the Racah polynomials in the first graph of Fig. 2, see also Fig. 4. The method of proof is by examining continuous dependence on the parameters in the coefficients for the three-term recurrence relation of the monic orthogonal polynomials.

The present paper extends the results of [17] such that the whole Askey scheme is covered. It is helpful to view this in a geometric way as several four-manifolds with coordinates coming from the parameters for the Racah or Wilson polynomials. Here we have one four-manifold for the Racah polynomials and two distinct fourmanifolds for the Wilson polynomials. We should consider these four-manifolds together with boundary, not just a boundary of codimension 1, but boundary parts of all dimensions $0,1,2,3$. Such manifolds (in arbitrary finite dimension) are known as manifolds with corners, introduced by Cerf [5] and Douady [10]. The various boundary parts will correspond with families lying below the top level in the Askey scheme. It turns out that for each of the two Wilson manifolds (see Fig. 3) one reparametrization is sufficient. But in order to cover the Racah polynomials and everything below, three reparametrizations (local charts) are needed, which partially overlap. See Fig. 2 for a graphical representation of what is covered by each of the three charts.

The detailed results are quite computational. In order to obtain them, I made heavy use of the computer algebra program Mathematica ${ }^{\circledR}$. The Mathematica notebooks with the computations will be available on the web, see http://www.science.uva.nl/ thk/art/.

Various papers have appeared about asymptotics in connection with the Askey scheme, see, for instance, [8, 9, 11-14, 19, 21, 23]. Limits are a very special case of asymptotics. The technique of the present paper for obtaining limits using the threeterm recurrence relation may be also relevant for further asymptotics.

Another potential application of this paper would be in the problem to identify some explicit system of orthogonal polynomials when the explicit coefficients in its three-term recurrence relation are given. In a joint work by the author with Swarttouw, a Maple ${ }^{\circledR}$ procedure called rec2ortho was written which can do this job for polynomials in the Askey scheme up to the 2-parameter level (Jacobi, Meixner, 
etc.), see http://www.science.uva.nl/ thk/art/software/rec2ortho/. The underlying algorithm is not very conceptual. It is based on a case by case analysis of the structure of the coefficients in the recurrence relations for the various families in the Askey scheme. Another approach, more conceptual but not yet covering everything, was proposed and implemented by Koepf and Schmersau [16]. In view of the present paper, the approach of rec2ortho, which has to deal with 13 families in order to cover the whole Askey scheme, might be adapted by looking for a match with one of the five cases in Sects. 7 and 8.

The contents of this paper are as follows. Sects. 2 and 3 illustrate the general principle for the case of the classical orthogonal polynomials. Manifolds with corners are briefly introduced in Sect. 4. The Askey scheme is presented in Sect. 5. Next a summary of the results, including the various parts of the Askey scheme covered by the five local charts, is given in Sect. 6. The detailed results are in Sects. 7 and 8, for the Racah and Wilson manifolds, respectively. In conclusion, Sect. 9 discusses the results and formulates work yet to be done.

\section{The general principle}

Consider the classical orthogonal polynomials (see Chihara [6, Chap. V, Sect. 2]) as monic polynomials $p_{n}(x)=x^{n}+$ terms of degree less than $n$ :

- Jacobi polynomials $p_{n}^{(\alpha, \beta)}(x)$ with weight function $(1-x)^{\alpha}(1+x)^{\beta}$ on $(-1,1)$.

- Laguerre polynomials $\ell_{n}^{\alpha}(x)$ with weight function $e^{-x} x^{\alpha}$ on $(0, \infty)$.

- Hermite polynomials $h_{n}(x)$ with weight function $e^{-x^{2}}$ on $(-\infty, \infty)$.

They are connected by limit relations (see [15, Chap. 2]):

$$
\begin{aligned}
\lim _{\beta \rightarrow \infty}(-\beta / 2)^{n} p_{n}^{(\alpha, \beta)}(1-2 x / \beta) & =\ell_{n}^{\alpha}(x), \\
\lim _{\alpha \rightarrow \infty} \alpha^{n / 2} p_{n}^{(\alpha, \alpha)}\left(x / \alpha^{1 / 2}\right) & =h_{n}(x), \\
\lim _{\alpha \rightarrow \infty}(2 \alpha)^{-n / 2} \ell_{n}^{\alpha}\left((2 \alpha)^{1 / 2} x+\alpha\right) & =h_{n}(x) .
\end{aligned}
$$

The limit relation (2.1) is immediate from the explicit expressions of Jacobi and Laguerre polynomials as terminating hypergeometric series (see [15, (1.8.1), (1.8.4), (1.11.1), (1.11.4)]):

$$
\begin{gathered}
p_{n}^{(\alpha, \beta)}(1-2 x)=\frac{2^{n}(\alpha+1)_{n}}{(n+\alpha+\beta+1)_{n}}{ }_{2} F_{1}\left(\begin{array}{c}
-n, n+\alpha+\beta+1 \\
\alpha+1
\end{array} ; x\right), \\
\ell_{n}^{\alpha}(x)=(-1)^{n}(\alpha+1)_{n 1} F_{1}\left(\begin{array}{c}
-n \\
\alpha+1
\end{array} ; x\right) .
\end{gathered}
$$

Then (2.2) is equivalent by quadratic transformations to the cases $\alpha= \pm \frac{1}{2}$ of (2.1).

The limit relation (2.3), first observed in 1939 by Palamà [20] and by Toscano [24], cannot be easily obtained from explicit power series. An approach to prove (2.3) by taking limits of the corresponding weight functions was given by Askey [3]. 
His approach works also for (2.1) and (2.2). Indeed, the limit relations for the weight functions corresponding to (2.1)-(2.3) are:

$$
\begin{aligned}
\lim _{\beta \rightarrow \infty} x^{\alpha}(1-x / \beta)^{\beta} & =x^{\alpha} e^{-x}, \\
\lim _{\alpha \rightarrow \infty}\left(1-x^{2} / \alpha\right)^{\alpha} & =e^{-x^{2}}, \\
\lim _{\alpha \rightarrow \infty}\left(1+(2 / \alpha)^{1 / 2} x\right)^{\alpha} e^{-(2 \alpha)^{1 / 2} x} & =e^{-x^{2}} .
\end{aligned}
$$

The limits (2.4) and (2.5) are standard limits, while (2.6), observed by Askey [3], is an easy exercise. What we, in fact, need in order to conclude rigorously that (2.1)(2.3) follow from (2.4)-(2.6) is not just the pointwise limits for the corresponding weight functions, but the limits for the corresponding moments:

Proposition 2.1 Let there be given monic orthogonal polynomials $\left(p_{n}\right)_{n=0,1,2, \ldots}$ with respect to an orthogonality measure having moments $\mu_{n}(n=0,1,2, \ldots)$ and, for $\alpha>0$, monic orthogonal polynomials $\left(p_{n}^{\alpha}\right)_{n=0,1,2, \ldots}$ with respect to an orthogonality measure having moments $\mu_{n}^{\alpha}(n=0,1,2, \ldots)$. If $\lim _{\alpha \rightarrow \infty} \mu_{n}^{\alpha}=\mu_{n}$ for all $n$ then $\lim _{\alpha \rightarrow \infty} p_{n}^{\alpha}(x)=p_{n}(x)$ for all $n$.

Proof Use the fact that

$$
p_{n}(x)=\left(\operatorname{det}\left(\mu_{i+j}\right)_{i, j=0,1, \ldots, n-1}\right)^{-1}\left|\begin{array}{ccccc}
\mu_{0} & \mu_{1} & \mu_{2} & \ldots & \mu_{n} \\
\mu_{1} & \mu_{2} & \mu_{3} & \ldots & \mu_{n+1} \\
\cdots & \cdots & \cdots & \cdots & \cdots \\
\mu_{n-1} & \mu_{n} & \mu_{n+1} & \cdots & \mu_{2 n-1} \\
1 & x & x^{2} & \cdots & x^{n}
\end{array}\right|
$$

(see $[22,(2.2 .6)])$, and similarly for $p_{n}^{\alpha}(x)$.

In order to conclude from pointwise limits of weight functions as in (2.4)-(2.6) that the corresponding limits for the moments hold, one needs Lebesgue's dominated convergence theorem. The relevant inequalities corresponding to (2.4)-(2.6) are:

$$
\begin{aligned}
x^{\alpha}(1-x / \beta)^{\beta} & \leq x^{\alpha} e^{-x}, \\
\left(1-x^{2} / \alpha\right)^{\alpha} & \leq e^{-x^{2},} \\
\left(1+(2 / \alpha)^{1 / 2} x\right)^{\alpha} e^{-(2 \alpha)^{1 / 2} x} & \leq \begin{cases}e^{-x^{2}} & \left(-(\alpha / 2)^{1 / 2}<x \leq 0\right), \\
(1+2 x) e^{-x} & \left(x \geq 0, \alpha \geq \frac{1}{2}\right) .\end{cases}
\end{aligned}
$$

In this paper, I want to advertise another method to prove limit formulas of the above type for orthogonal polynomials, namely by using the three-term recurrence relation. The celebrated Favard theorem (see Chihara [6, Chap. I, Theorem 4.4]) states that $\left\{p_{n}\right\}_{n=0,1,2, \ldots}$ is a system of monic orthogonal polynomials with respect to a positive orthogonality measure if and only if a recurrence relation

$$
x p_{n}(x)=p_{n+1}(x)+B_{n} p_{n}(x)+C_{n} p_{n-1}(x), \quad n=1,2, \ldots,
$$




$$
\begin{aligned}
x p_{0}(x) & =p_{1}(x)+B_{0} p_{0}(x), \\
p_{0}(x) & =1,
\end{aligned}
$$

is valid with $C_{n}>0$ and $B_{n}$ real. Below, when we will give the recurrence relation (2.7) with explicit coefficients $B_{n}$ and $C_{n}$ depending analytically on $n$, then we will silently assume that it also implies the case $n=0$, i.e., (2.8). Just take $B_{n}$ for $n=0$ and omit the term $C_{n} p_{n-1}(x)$ for $n=0$.

If the coefficients $B_{n}$ and $C_{n}$ are given then $p_{n}$ is completely determined by this recurrence relation. In particular, if $B_{n}$ and $C_{n}$ continuously depend on some parameter $\lambda$ then $p_{n}(x)$ will also continuously depend on $\lambda$. For example, monic Hermite polynomials $h_{n}$ satisfy the recurrence relation

$$
x h_{n}(x)=h_{n+1}(x)+\frac{1}{2} n h_{n-1}(x),
$$

and monic Laguerre polynomials $\ell_{n}^{\alpha}$ satisfy the recurrence relation

$$
x \ell_{n}^{\alpha}(x)=p_{n+1}(x)+(2 n+\alpha+1) \ell_{n}^{\alpha}(x)+n(n+\alpha) \ell_{n-1}^{\alpha}(x) .
$$

Now consider rescaled monic Laguerre polynomials

$$
p_{n}(x)=p_{n}(x ; \alpha, \rho, \sigma):=\rho^{n} \ell_{n}^{\alpha}\left(\rho^{-1} x-\sigma\right) .
$$

By (2.10), these satisfy the recurrence relation

$$
x p_{n}(x)=p_{n+1}(x)+\rho(2 n+\alpha+1+\sigma) p_{n}(x)+\rho^{2} n(n+\alpha) p_{n-1}(x) .
$$

We want to rescale in such a way that, as $\alpha \rightarrow \infty, p_{n}(x)$ will tend to $h_{n}(x)$. It is easy to see how to do this when we compare (2.9) and (2.11). Put $\rho:=(2 \alpha)^{-1 / 2}, \sigma:=-\alpha$. Then (2.11) becomes

$$
x p_{n}(x)=p_{n+1}(x)+(2 \alpha)^{-1 / 2}(2 n+1) p_{n}(x)+\frac{n(n+\alpha)}{2 \alpha} p_{n-1}(x) .
$$

The recurrence coefficients now tend to 0 (resp., $n / 2$ ) as $\alpha \rightarrow \infty$. Hence $p_{n}(x) \rightarrow$ $h_{n}(x)$ as $\alpha \rightarrow \infty$, i.e., we have recovered (2.3).

\section{Uniform limit of Jacobi polynomials}

It is now natural to conjecture that we might also make these limit transitions in the parameter plane in a more uniform way, i.e., to make such a rescaling of the Jacobi polynomials that they depend continuously on $(\alpha, \beta)$ in the extended parameter plane and reduce to (possibly rescaled) Laguerre and Hermite polynomials on the boundary lines and boundary vertex at infinity, respectively. For this purpose, we consider Jacobi polynomials with arbitrary rescaling:

$$
p_{n}(x):=\rho^{n} p_{n}^{(\alpha, \beta)}\left(\rho^{-1} x-\sigma\right) .
$$


These polynomials satisfy recurrence relations (2.7) with (see $[15,(1.8 .4)])$ :

$$
\begin{aligned}
C_{n} & :=\rho^{2} \frac{4 n(n+\alpha)(n+\beta)(n+\alpha+\beta)}{(2 n+\alpha+\beta-1)(2 n+\alpha+\beta)^{2}(2 n+\alpha+\beta+1)} \\
& =\frac{\rho^{2} \alpha \beta}{(\alpha+\beta)^{3}} \frac{4 n(1+n / \alpha)(1+n / \beta)(1+n /(\alpha+\beta))}{(1+(2 n-1) /(\alpha+\beta))(1+2 n /(\alpha+\beta))^{2}(1+(2 n+1) /(\alpha+\beta))}
\end{aligned}
$$

and

$$
\begin{aligned}
B_{n} & :=\rho\left(\frac{\beta^{2}-\alpha^{2}}{(2 n+\alpha+\beta)(2 n+\alpha+\beta+2)}+\sigma\right) \\
& =\rho\left(\frac{\beta-\alpha}{\beta+\alpha} \frac{1}{1+2 n /(\alpha+\beta)} \frac{1}{1+(2 n+2) /(\alpha+\beta)}+\sigma\right) .
\end{aligned}
$$

From (3.2) we see that the choice

$$
\rho:=\frac{(\alpha+\beta)^{3 / 2}}{\alpha^{1 / 2} \beta^{1 / 2}}
$$

makes $C_{n}$ continuous in $(\alpha, \beta)$ on the extended parameter plane. Next we see from (3.3) that the choice

$$
\sigma:=\frac{\alpha-\beta}{\alpha+\beta}
$$

makes $B_{n}$ continuous in $(\alpha, \beta)$ (extended) as well. Indeed, we can now rewrite

$$
\begin{aligned}
C_{n} & =\frac{4 n(1+n / \alpha)(1+n / \beta)(1+n /(\alpha+\beta))}{(1+(2 n-1) /(\alpha+\beta))(1+2 n /(\alpha+\beta))^{2}(1+(2 n+1) /(\alpha+\beta))}, \\
B_{n} & =\frac{\beta^{-1}-\alpha^{-1}}{\left(\alpha^{-1}+\beta^{-1}\right)^{1 / 2}} \frac{4 n+2+4 n(n+1) /(\alpha+\beta)}{(1+2 n /(\alpha+\beta))(1+(2 n+2) /(\alpha+\beta))},
\end{aligned}
$$

which are continuous in $\left(\alpha^{-1}, \beta^{-1}\right)$ for $\alpha^{-1}, \beta^{-1} \geq 0$.

As a result we can consider the $\left(\alpha^{-1}, \beta^{-1}\right)$-parameter plane. For $\alpha^{-1}, \beta^{-1}>0$, we have the rescaled Jacobi polynomials (3.1) with $\rho$ and $\sigma$ given by (3.4) and (3.5). These polynomials extend continuously to the closure $\left\{\left(\alpha^{-1}, \beta^{-1}\right) \mid \alpha^{-1}, \beta^{-1} \geq 0\right\}$. On the boundary lines $\left\{\left(\alpha^{-1}, 0\right) \mid \alpha^{-1}>0\right\}$ and $\left\{\left(0, \beta^{-1}\right) \mid \beta^{-1}>0\right\}$, these polynomials, in view of (2.11), become rescaled Laguerre polynomials

$$
\begin{aligned}
& \rho^{n} \ell_{n}^{\alpha}\left(\rho^{-1} x-\sigma\right) \quad \text { with } \rho=-2 \alpha^{-1 / 2}, \sigma=-\alpha, \\
& \text { resp., } \rho=2 \beta^{-1 / 2}, \sigma=-\beta .
\end{aligned}
$$

On the boundary vertex $(0,0)$, the polynomials, in view of $(2.9)$, become rescaled Hermite polynomials

$$
\rho^{n} h_{n}\left(\rho^{-1} x\right) \quad \text { with } \rho=2^{3 / 2} \text {. }
$$


Remark 3.1 Our limit

$$
\lim _{\alpha, \beta \rightarrow \infty} \rho^{n} p_{n}^{(\alpha, \beta)}\left(\rho^{-1} x-\sigma\right)=2^{3 n / 2} h_{n}\left(2^{-3 n / 2} x\right)
$$

with $\rho, \sigma$ given by (3.4), (3.5) implies the limit given in [11, Sect. 2.6.4].

\section{Manifolds with corners}

The closed subset

$$
\left\{\left(\alpha^{-1}, \beta^{-1}\right) \in \mathbb{R}^{2} \mid \alpha^{-1}, \beta^{-1} \geq 0\right\}
$$

of the $\left(\alpha^{-1}, \beta^{-1}\right)$-parameter plane considered at the end of Sect. 3 is a prototype of a so-called manifold with corners. More generally, define

$$
\mathbb{R}_{(q)}^{n}:=\left\{\left(x_{1}, \ldots, x_{n}\right) \in \mathbb{R}^{n} \mid x_{q+1}, \ldots, x_{n} \geq 0\right\} \quad(q=0,1, \ldots, n) .
$$

Manifolds with corners, introduced by Cerf [5] and Douady [10], are topological Hausdorff spaces which are locally homeomorphic to open subsets of spaces $\mathbb{R}_{(q)}^{n}$. The definition is analogous to the definition of an ordinary manifold, but there the local homeomorphisms only map onto open subsets of $\mathbb{R}^{n}$. Thus, for a manifold with corners denoted by $X$, we have charts $(U, \phi)$ such that $\phi: U \rightarrow \phi(U)$ is a homeomorphism from an open subset $U$ of $X$ onto an open subset $\phi(U)$ of some $\mathbb{R}_{(q)}^{n}$. If $(U, \phi)$ and $(V, \psi)$ are two charts on $X$ then $\psi \circ \phi^{-1}: \phi(U \cap V) \rightarrow \psi(U \cap V)$ must be a homeomorphism. If these maps extend on a larger open subset of $\mathbb{R}^{n}$ to $C^{k}$ diffeomorphisms (or $C^{\infty}$-diffeomorphisms, or analytic diffeomorphisms), then $X$ is called a $C^{k}$ (or $C^{\infty}$, or analytic) manifold with corners.

On the prototypical manifold with corners given by (4.1), each point can be associated with a system of orthogonal polynomials. The coefficients $C_{n}$ and $B_{n}$ given by (3.6) and (3.7) are continuous on this manifold, and thus the resulting orthogonal polynomials $p_{n}$ obtained from $C_{n}$ and $B_{n}$ by the recurrence relation (2.7) are continuous on the manifold. In the interior, on the boundary lines and on the boundary vertex these polynomials respectively become (rescaled) Jacobi, Laguerre and Hermite polynomials as described at the end of Sect. 3. Note, however, that $B_{n}$ given by (3.7) is not differentiable at the point $\left(\alpha^{-1}, \beta^{-1}\right)=(0,0)$.

\section{The Askey scheme}

The Askey scheme is given by Fig. 1. The various families of orthogonal polynomials mentioned here are all of classical type, i.e., the orthogonal polynomials $\left\{p_{n}\right\}_{n=0,1, \ldots}$ satisfy an equation of the form

$$
L p_{n}=\lambda_{n} p_{n},
$$

where $L$ is some second order operator (differential or difference) which does not depend on $n$. The arrows in the chart denote limit transitions between the various 
Fig. 1 Askey scheme

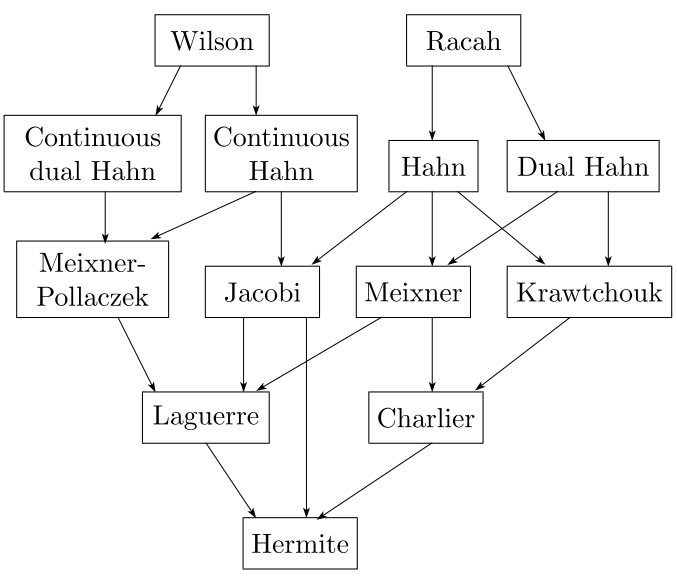

families. The number of additional parameters on which the polynomials depend decreases as we go further down in the chart. In the top row, there are 4 parameters. In each subsequent row, there is one parameter less. The Hermite polynomials in the bottom row no longer depend on parameters. The families in the left part of the chart consist of polynomials being orthogonal with respect to an absolutely continuous measure, while the ones displayed to the right of Hermite are orthogonal with respect to a discrete measure. In the case of Racah, Hahn, dual Hahn and Krawtchouk polynomials, the support of the measure has finite cardinality, say $N+1$, and we consider only polynomials up to degree $N$. All the polynomials in the Askey scheme have explicit expressions as hypergeometric functions, see [15, Chap. 1].

Racah polynomials (see [15, Sect. 1.2]) are defined by

$$
\begin{aligned}
& R_{n}(y(y+\gamma+\delta+1) ; \alpha, \beta, \gamma, \delta) \\
& \quad:={ }_{4} F_{3}\left(\begin{array}{c}
-n, n+\alpha+\beta+1,-y, y+\gamma+\delta+1 \\
\alpha+1, \beta+\delta+1, \gamma+1
\end{array} ;\right),
\end{aligned}
$$

where $\gamma+1=-N$ and $n=0,1, \ldots, N$. Then we write the monic Racah polynomials as

$$
\begin{aligned}
r_{n}(x) & =r_{n}(x ; \alpha, \beta,-N-1, \delta) \\
& :=\frac{(\alpha+1)_{n}(\beta+\delta+1)_{n}(-N)_{n}}{(n+\alpha+\beta+1)_{n}} R_{n}(x ; \alpha, \beta,-N-1, \delta) .
\end{aligned}
$$

Wilson polynomials (see [15, Sect. 1.1]) are defined by

$$
\begin{aligned}
& W_{n}\left(y^{2} ; a, b, c, d\right) \\
& \quad:=(a+b)_{n}(a+c)_{n}(a+d)_{n} \\
& \quad \times{ }_{4} F_{3}\left(\begin{array}{c}
-n, n+a+b+c+d-1, a+i x, a-i x \\
a+b, a+c, a+d
\end{array}\right) .
\end{aligned}
$$


Then we write the monic Wilson polynomials as

$$
w_{n}(x)=w_{n}(x ; a, b, c, d):=\frac{(-1)^{n}}{(n+a+b+c+d-1)_{n}} W_{n}(x ; a, b, c, d) .
$$

The following list gives the notation to be used in this paper for the monic orthogonal polynomials in the other families (apart from Racah and Wilson) in the Askey scheme. In each case, the monic polynomials are expressed in terms of the polynomials in usual notation and normalization as given under the heading "Definition" in each subsection of [15, Chap. 1]. The constants occurring in the definitions of the monic polynomials are taken from the formulas for $p_{n}$ under the heading "Normalized recurrence relation" in each subsection of [15, Chap. 1]:

Hahn [15, Sect. 1.5],

$$
q_{n}(x ; \alpha, \beta, N):=\frac{(\alpha+1)_{n}(-N)_{n}}{(n+\alpha+\beta+1)_{n}} Q_{n}(x ; \alpha, \beta, N) ;
$$

Dual Hahn [15, Sect. 1.6],

$$
r_{n}^{\mathrm{DH}}(x ; \gamma, \delta, N):=(\gamma+1)_{n}(-N)_{n} R_{n}(x ; \gamma, \delta, N) ;
$$

Jacobi [15, Sect. 1.8],

$$
p_{n}^{(\alpha, \beta)}(x):=\frac{2^{n} n !}{(n+\alpha+\beta+1)_{n}} P_{n}^{(\alpha, \beta)}(x) ;
$$

Meixner [15, Sect. 1.9],

$$
m_{n}(x ; \beta, c):=(\beta)_{n}\left(\frac{c}{c-1}\right)^{n} M_{n}(x ; \beta, c) ;
$$

Krawtchouk [15, Sect. 1.10],

$$
k_{n}(x ; p, N):=(-N)_{n} p^{n} K_{n}(x ; p, N) ;
$$

Laguerre [15, Sect. 1.11],

$$
\ell_{n}^{\alpha}(x):=(-1)^{n} n ! L_{n}^{(\alpha)}(x)
$$

Charlier [15, Sect. 1.12],

$$
c_{n}(x ; a):=(-1)^{n} a^{n} C_{n}(x ; a) ;
$$

Hermite [15, Sect. 1.13],

$$
h_{n}(x):=2^{-n} H_{n}(x) ;
$$

Continuous Hahn [15, Sect. 1.4],

$$
p_{n}^{\mathrm{CH}}(x ; a, b, c, d):=\frac{n !}{(n+a+b+c+d-1)_{n}} p_{n}(x ; a, b, c, d) ;
$$


Continuous dual Hahn [15, Sect. 1.3],

$$
s_{n}(x ; a, b, c):=(-1)^{n} S_{n}(x ; a, b, c) ;
$$

Meixner-Pollaczek [15, Sect. 1.7],

$$
p_{n}^{(\lambda)}(x ; \phi):=\frac{n !}{(2 \sin \phi)^{n}} P_{n}^{(\lambda)}(x ; \phi) .
$$

\section{Uniform limits in the Askey scheme}

The monic Racah polynomials (5.2) satisfy (see [15, (1.2.4)]) the three-term recurrence relation

$$
x r_{n}(x)=r_{n+1}(x)+B_{n} r_{n}(x)+C_{n} r_{n-1}(x),
$$

where $B_{n}=a_{n}+c_{n}, C_{n}=a_{n-1} c_{n}$ with

$$
\begin{aligned}
a_{n} & :=\frac{(n+\alpha+1)(n+\alpha+\beta+1)(n+\beta+\delta+1)(N-n)}{(2 n+\alpha+\beta+1)(2 n+\alpha+\beta+2)}, \\
c_{n}: & =\frac{n(n+\alpha+\beta+N+1)(\delta-\alpha-n)(n+\beta)}{(2 n+\alpha+\beta)(2 n+\alpha+\beta+1)} .
\end{aligned}
$$

The Racah polynomials $\left\{r_{n}\right\}_{n=0,1, \ldots, N}$ will be orthogonal with respect to certain positive weights on some set of $N+1$ points in $\mathbb{R}$ iff $C_{n}>0$ for $n=1,2, \ldots, N$. A sufficient condition for this is that the parameters satisfy the inequalities $\alpha, \beta>-1$, $\delta>\alpha+N$ and $N>0$ ( $N$ integer). It will be convenient to restrict to the smaller parameter region

$$
\alpha, \beta>0, \quad N>1, \quad \delta>\alpha+N,
$$

and to drop the assumption that $N$ is integer. All limits to be considered will be in this parameter region.

Each point in the four-dimensional $(\alpha, \beta, \delta, N)$-space satisfying (6.1) thus corresponds with a system of Racah polynomials being orthogonal with respect to a positive measure (if $N$ is moreover integer). This defines an open subset $X_{0}$ of $\mathbb{R}^{4}$. Analogously to what we did in Sect. 3 for an open part of $(\alpha, \beta)$-parameter space corresponding with Jacobi polynomials, we want to find suitable local coordinates (charts $(U, \phi))$ in the four-dimensional Racah parameter space $X_{0}$ and suitable rescalings of the Racah polynomials which depend on these local coordinates such that the resulting coefficients $B_{n}$ and $C_{n}$ in the recurrence relation have a continuous extension to the boundary of $\phi(U)$, and such that their restriction to (parts of certain dimension of) this boundary corresponds to certain arrows lying somewhere under the Racah box in Fig. 1. $N^{-1}$ should be a monomial in these coordinates. Thus, on approaching the boundary, $N^{-1}$ will be either fixed or it will tend to 0 . As a consequence, it is only a minor shortcoming in our set-up that $N$ is discrete.

It will turn out that in this way we can extend the Racah parameter space $X_{0}$ to a manifold with corners $X$ (the Racah manifold) covering everything which lies below 

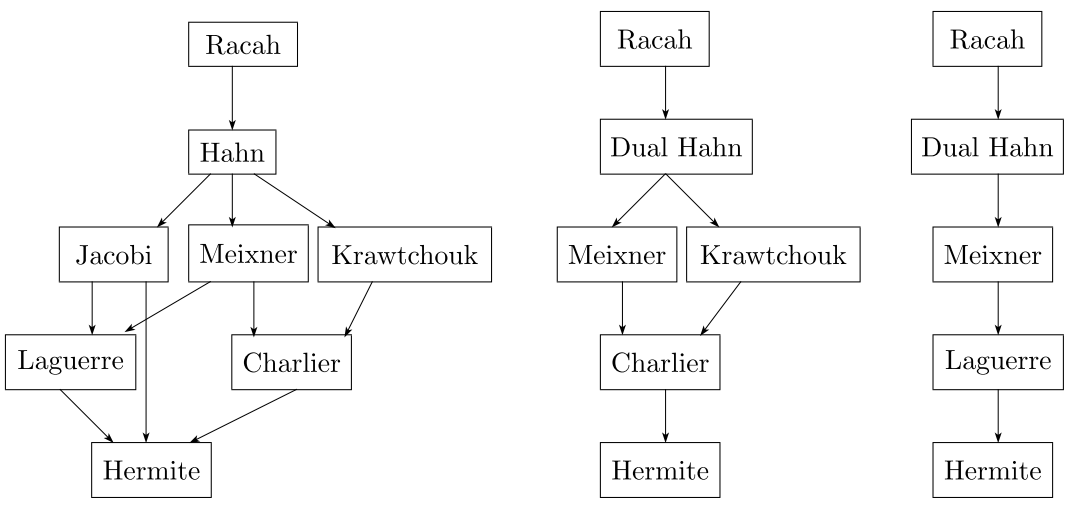

Fig. 2 Parts of the Racah manifold covered by the three specific charts

the Racah box in the Askey scheme if we use three local charts. The three parts of the Askey scheme which are respectively covered by these charts are given in Fig. 2.

The monic Wilson polynomials (5.4) satisfy (see [15, (1.1.5)]) the three-term recurrence relation

$$
x w_{n}(x)=w_{n+1}(x)+B_{n} w_{n}(x)+C_{n} w_{n-1}(x),
$$

where $B_{n}=a_{n}+c_{n}-a^{2}, C_{n}=a_{n-1} c_{n}$ with

$$
\begin{aligned}
a_{n} & :=\frac{(n+a+b+c+d-1)(n+a+b)(n+a+c)(n+a+d)}{(2 n+a+b+c+d-1)(2 n+a+b+c+d)}, \\
c_{n} & :=\frac{n(n+b+c-1)(n+b+d-1)(n+c+d-1)}{(2 n+a+b+c+d-2)(2 n+a+b+c+d-1)} .
\end{aligned}
$$

Then $B_{n}$ and $C_{n}$ turn out to be symmetric in $a, b, c, d$, and therefore $w_{n}(x ; a, b, c, d)$ is symmetric in $a, b, c, d$. The polynomials $\left\{w_{n}\right\}_{n=0,1,2, \ldots}$ will be orthogonal with respect to a certain positive measure on $\mathbb{R}$ iff $C_{n}>0$ for $n=1,2, \ldots$. This, in its turn, is equivalent to one of the following three cases:

1. $a, b, c, d$ are non-real and occur in complex conjugate pairs with positive real parts.

2. Two of the four parameters $a, b, c, d$ are non-real and form a complex conjugate pair with positive real part. The other two parameters are real and their sum is positive.

3. $a, b, c, d$ are real and $C_{n}>0$ for $n=1,2, \ldots$

Limits of Wilson polynomials in the Askey scheme occur in all these three cases, see Fig. 3. In case 1, we have a limit to the continuous Hahn polynomials and to everything below the continuous Hahn polynomials. In case 2, we have a limit to the continuous dual Hahn polynomials and to everything below the continuous dual Hahn polynomials. To each of these two cases will correspond one manifold with corners. In case 3 , we also have a limit to the continuous dual Hahn polynomials, but 

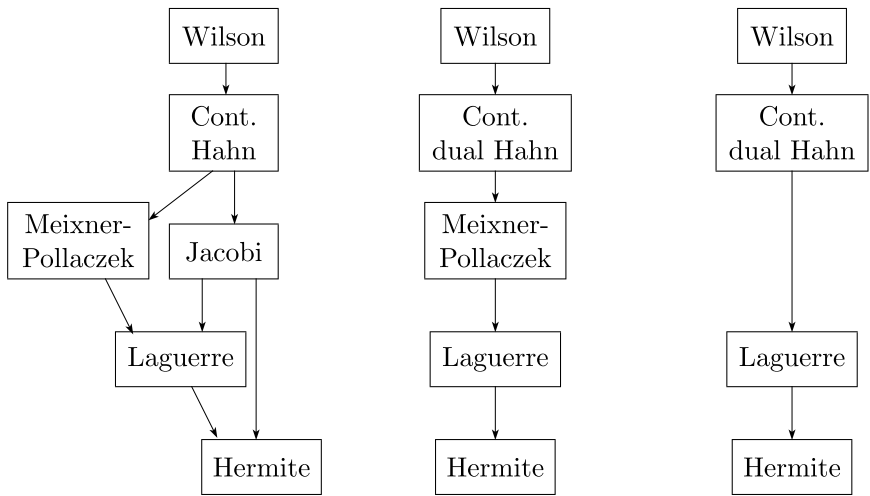

Fig. 3 Limits of Wilson polynomials: on the left parameters in two complex conjugate pairs; in the middle one complex conjugate pair and two real; on the right all parameters real

from there a limit to the two-parameter level seems to be missing; one rather goes to Laguerre as follows:

$$
\lim _{c \rightarrow \infty} c^{-n} s_{n}(c x ; a, b, c)=\ell_{n}^{a+b-1}(x) .
$$

This is straightforward from $[15,(1.3 .1),(1.11 .1)]$ and the formulas for the monic continuous dual Hahn and Laguerre polynomials in Sect. 5. The limit (6.2) is missing in [1, Appendix] and [15, Sect. 2.3].

It will turn out that one chart is sufficient in cases 1 and 2 in order to cover everything which is below the Wilson box. I did not try to find a chart for case 3 . If

The following simple observation will be continuously used in the next sections.

$$
x p_{n}(x)=p_{n+1}(x)+B_{n} p_{n}(x)+C_{n} p_{n-1}(x),
$$

and if

$$
q_{n}(x):=\rho^{n} p_{n}\left(\rho^{-1} x-\sigma\right)
$$

then

$$
x q_{n}(x)=q_{n+1}(x)+\rho\left(B_{n}+\sigma\right) q_{n}(x)+\rho^{2} C_{n} q_{n-1}(x) .
$$

\section{The Racah manifold}

In this section, I will present in detail the three charts covering the Racah manifold, as introduced in Sect. 6, and successively corresponding to the three graphs in Fig. 2.

\subsection{From Racah to Hermite along Hahn}

Here we will see the chart for the Racah manifold corresponding to the first graph in Fig. 2. The monic Racah polynomial $r_{n}$ is given by (5.2). For $t_{1}, t_{2}, t_{3}, t_{4}>0$ and 
$t_{1} t_{3}<1, t_{2} t_{4}<1$ put

$$
p_{n}(x)=p_{n}\left(x ; t_{1}, t_{2}, t_{3}, t_{4}\right):=\rho^{n} r_{n}\left(\rho^{-1} x-\sigma ; \alpha, \beta,-N-1, \delta\right),
$$

where

$$
\begin{aligned}
\alpha & =\frac{1}{t_{1}}, \quad \beta=\frac{1}{t_{1} t_{2}}, \quad N=\frac{1}{t_{2} t_{4}}, \quad \delta=\frac{1+t_{2} t_{3} t_{4}}{t_{1} t_{2} t_{3} t_{4}}, \\
\rho & =\frac{t_{1} t_{2}\left(1+t_{2}\right)^{3 / 2} t_{3} t_{4}^{2}}{\left(t_{1}+t_{4}+t_{2} t_{4}\right)^{1 / 2}\left(1+\left(1+t_{2}\right) t_{3} t_{4}\right)^{1 / 2}}, \\
\sigma & =-\frac{\left(1+t_{1}\right)\left(1+\left(1+t_{2}+t_{1} t_{2}\right) t_{3} t_{4}\right)}{t_{1} t_{2}\left(1+t_{2}+2 t_{1} t_{2}\right) t_{3} t_{4}^{2}} .
\end{aligned}
$$

Then the inequalities for $t_{1}, t_{2}, t_{3}, t_{4}$ are equivalent to (6.1), and the recurrence relation (6.3) holds with

$$
\begin{aligned}
B_{n}= & -\frac{n\left(1+t_{2}\right)^{3 / 2}\left(1+t_{2}+(n+1) t_{1} t_{2}\right)}{\left(1+t_{2}+2 t_{1} t_{2}\right)\left(1+t_{2}+2 n t_{1} t_{2}\right)\left(1+t_{2}+2(n+1) t_{1} t_{2}\right)\left(1+t_{3} t_{4}+t_{2} t_{3} t_{4}\right)^{1 / 2}} \\
& \times\left(2 n t_{1} t_{2} t_{3} t_{4}^{2}\left(1+t_{2}+(n+1) t_{1} t_{2}\right)\left(1+t_{2}+2 t_{1} t_{2}\right)\right. \\
& +t_{2} t_{3} t_{4}^{2}\left(1+t_{1}\right)\left(1+t_{2}\right)\left(1+t_{2}+2 t_{1} t_{2}\right)-t_{4}\left(1-t_{2}^{2}\right)\left(1+t_{1} t_{3}\right) \\
& \left.-2 t_{1}\left(1-t_{2}\right)\left(1+t_{2} t_{4}\right)\right) /\left(t_{1}+t_{4}+t_{2} t_{4}\right)^{1 / 2}, \\
C_{n}= & \frac{\left(1+t_{2}+n t_{1} t_{2}\right)\left(1+(1-n) t_{2} t_{4}\right)\left(1-n t_{1} t_{2} t_{3} t_{4}\right)\left(1+t_{3} t_{4}+t_{2} t_{3} t_{4}+n t_{1} t_{2} t_{3} t_{4}\right)}{\left(1+t_{2}+(2 n-1) t_{1} t_{2}\right)\left(1+t_{2}+2 n t_{1} t_{2}\right)^{2}\left(1+t_{2}+(2 n+1) t_{1} t_{2}\right)} \\
& \times n\left(1+n t_{1}\right)\left(1+n t_{1} t_{2}\right) \frac{\left(1+t_{2}\right)^{3}}{1+t_{3} t_{4}+t_{2} t_{3} t_{4}}\left(1+(n+1) \frac{t_{1} t_{2} t_{4}}{t_{1}+t_{4}+t_{2} t_{4}}\right) .
\end{aligned}
$$

Note that $B_{n}$ and $C_{n}$, as functions of $t_{1}, t_{2}, t_{3}, t_{4}>0$, can be uniquely extended to continuous (but not differentiable) functions of $t_{1}, t_{2}, t_{3}, t_{4} \geq 0$. Indeed, for $B_{n}$ observe that if $t_{1}, t_{2}, t_{3}, t_{4}>0$ then

$$
0 \leq \frac{t_{1}}{\left(t_{1}+t_{4}+t_{2} t_{4}\right)^{1 / 2}} \leq t_{1}^{1 / 2}, \quad 0 \leq \frac{t_{4}}{\left(t_{1}+t_{4}+t_{2} t_{4}\right)^{1 / 2}} \leq t_{4}^{1 / 2} .
$$

For $C_{n}$ observe that if $t_{1}, t_{2}, t_{3}, t_{4}>0$ then

$$
0 \leq \frac{t_{1} t_{2} t_{4}}{t_{1}+t_{4}+t_{2} t_{4}} \leq \frac{\frac{1}{4}\left(t_{1}+t_{2} t_{4}\right)^{2}}{t_{1}+t_{2} t_{4}}=\frac{1}{4}\left(t_{1}+t_{2} t_{4}\right) .
$$

We now set one or more of the $t_{1}, t_{2}, t_{3}, t_{4}$ equal to zero in (7.3) and (7.4), and then compare the resulting recurrence relation (6.3) with the various normalized recurrence relations in [15, Chap. 1]. In each case, we find a match with one of these normalized recurrence relations, after rescaling as in (6.4) for some special $\rho, \sigma$. Then the explicitly obtained family in the Askey scheme satisfying this recurrence relation will be equal to the corresponding parameter restriction of the polynomial 
in (7.1). We obtain the following results, using the notation for monic polynomials in the Askey scheme as given at the end of Sect. 5:

Hahn,

$$
\begin{aligned}
& p_{n}\left(x ; t_{1}, t_{2}, 0, t_{4}\right)=\rho^{n} q_{n}\left(\rho^{-1} x-\sigma ; \alpha, \beta, N\right), \\
& \alpha=\frac{1}{t_{1}}, \beta=\frac{1}{t_{1} t_{2}}, N=\frac{1}{t_{2} t_{4}}, \\
& \rho=\frac{\left(1+t_{2}\right)^{3 / 2} t_{4}}{\left(t_{1}+t_{4}+t_{2} t_{4}\right)^{1 / 2}}, \sigma=-\frac{1+t_{1}}{\left(1+t_{2}+2 t_{1} t_{2}\right) t_{4}} ;
\end{aligned}
$$

Jacobi,

$$
\begin{gathered}
p_{n}\left(x ; t_{1}, t_{2}, t_{3}, 0\right)=p_{n}\left(x ; t_{1}, t_{2}, 0,0\right)=\rho^{n} p_{n}^{(\alpha, \beta)}\left(\rho^{-1} x-\sigma\right), \\
\alpha=\frac{1}{t_{1}}, \beta=\frac{1}{t_{1} t_{2}}, \rho=-\frac{\left(1+t_{2}\right)^{3 / 2}}{2 t_{1}^{1 / 2} t_{2}}, \sigma=\frac{-1+t_{2}}{1+t_{2}+2 t_{1} t_{2}} ;
\end{gathered}
$$

Meixner,

$$
\begin{aligned}
& p_{n}\left(x ; t_{1}, 0, t_{3}, t_{4}\right)=p_{n}\left(x ; t_{1}, 0,0, \frac{t_{4}\left(1-t_{1} t_{3}\right)}{1+t_{3} t_{4}}\right)=\rho^{n} m_{n}\left(\rho^{-1} x-\sigma ; \beta, c\right), \\
& \beta=\frac{1+t_{1}}{t_{1}}, c=\frac{t_{1}\left(1+t_{3} t_{4}\right)}{t_{1}+t_{4}}, \rho=\frac{\left(1-t_{1} t_{3}\right) t_{4}}{\left(t_{1}+t_{4}\right)^{1 / 2}\left(1+t_{3} t_{4}\right)^{1 / 2}}, \\
& \sigma=-\frac{\left(1+t_{1}\right)\left(1+t_{3} t_{4}\right)}{\left(1-t_{1} t_{3}\right) t_{4}}
\end{aligned}
$$

Krawtchouk,

$$
\begin{gathered}
p_{n}\left(x ; 0, t_{2}, t_{3}, t_{4}\right)=p_{n}\left(x ; 0, t_{2}\left(1+t_{3} t_{4}+t_{2} t_{3} t_{4}\right), 0, \frac{t_{4}}{1+t_{3} t_{4}+t_{2} t_{3} t_{4}}\right) \\
:=\rho^{n} k_{n}\left(\rho^{-1} x-\sigma ; p, N\right), \\
p=\frac{t_{2}\left(1+t_{3} t_{4}+t_{2} t_{3} t_{4}\right)}{\left(1+t_{2}\right)\left(1+t_{2} t_{3} t_{4}\right)}, N=\frac{1}{t_{2} t_{4}}, \\
\rho=\frac{t_{4}^{1 / 2}\left(1+t_{2}\right)\left(1+t_{2} t_{3} t_{4}\right)}{\left(1+t_{3} t_{4}+t_{2} t_{3} t_{4}\right)^{1 / 2}}, \sigma=-\frac{1+t_{3} t_{4}+t_{2} t_{3} t_{4}}{t_{4}\left(1+t_{2}\right)\left(1+t_{2} t_{3} t_{4}\right)} ;
\end{gathered}
$$

Laguerre,

$$
\begin{aligned}
& p_{n}\left(x ; t_{1}, 0, t_{3}, 0\right)=p_{n}\left(x ; t_{1}, 0,0,0\right)=\rho^{n} \ell_{n}^{(\alpha)}\left(\rho^{-1} x-\sigma\right), \\
& \alpha=\frac{1}{t_{1}}, \rho=t_{1}^{1 / 2}, \sigma=-\frac{1+t_{1}}{t_{1}}
\end{aligned}
$$




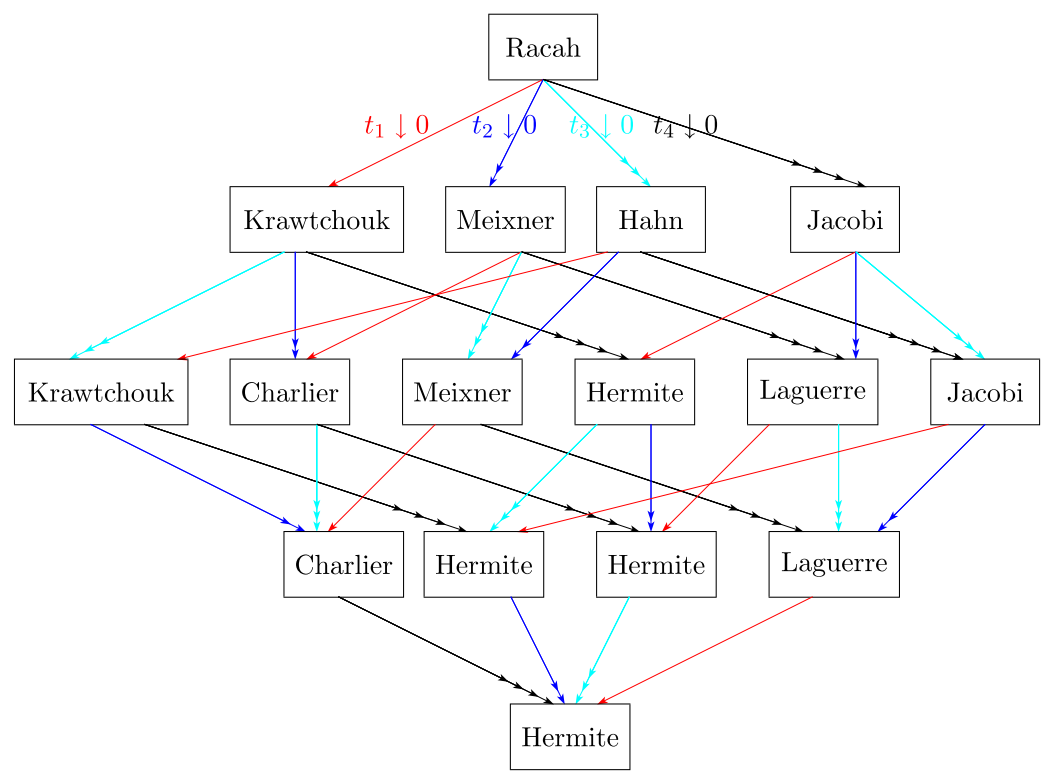

Fig. 4 The various parameter restrictions in the first chart for the Racah manifold

\section{Charlier,}

$$
\begin{gathered}
p_{n}\left(x ; 0,0, t_{3}, t_{4}\right)=p_{n}\left(x ; 0,0,0, \frac{t_{4}}{1+t_{3} t_{4}}\right)=\rho^{n} c_{n}\left(\rho^{-1} x-\sigma ; a\right), \\
a=\frac{1+t_{3} t_{4}}{t_{4}}, \rho=\frac{t_{4}^{1 / 2}}{\left(1+t_{3} t_{4}\right)^{1 / 2}}, \sigma=-\frac{1+t_{3} t_{4}}{t_{4}} ;
\end{gathered}
$$

Hermite,

$$
\begin{aligned}
p_{n}\left(x ; 0, t_{2}, t_{3}, 0\right) & =p_{n}\left(x ; 0, t_{2}, 0,0\right)=p_{n}\left(x ; 0,0, t_{3}, 0\right)=p_{n}(x ; 0,0,0,0) \\
& =\rho^{n} h_{n}\left(\rho^{-1} x-\sigma\right), \quad \rho=2^{1 / 2}, \sigma=0 .
\end{aligned}
$$

The various parameter restrictions of the polynomial (7.1) are summarized in Fig. 4. Note that Racah and Hahn occur in one box, most others in two boxes, and Hermite even in three boxes. For instance, we reach Jacobi from Racah by putting $t_{4}=0$, but then there is also no dependence on $t_{3}$, so we may put $t_{3}=0$ as well. The Meixner and Krawtchouk cases are slightly more complicated. For instance, we reach Meixner from Racah by putting $t_{2}=0$, but the resulting expression does not change if we replace $t_{3}, t_{4}$ by $0, t_{4}\left(1-t_{1} t_{3}\right)\left(1+t_{3} t_{4}\right)^{-1}$.

Remark 7.1 As a special case of the above results we have the following limit from Hahn to Hermite:

$$
\lim _{t_{1}, t_{4} \downarrow 0} p_{n}\left(x ; t_{1}, t_{2}, 0, t_{4}\right)=p_{n}\left(0, t_{2}, 0,0\right) .
$$


In view of (7.5) and (7.7), we can rewrite this limit as

$$
\lim _{a \rightarrow \infty} \rho^{n} q_{n}\left(\rho^{-1} x-\sigma ; a, a b, a N\right)=2^{n / 2} h_{n}\left(2^{-1 / 2} x\right),
$$

where

$$
\rho=\frac{(b+1)^{3 / 2}}{a^{1 / 2} b^{1 / 2} N^{1 / 2}(b+N+1)^{1 / 2}}, \quad \sigma=-\frac{a(a+1) N}{a b+a+2} .
$$

This limit is equivalent to the limit given in $[12,(16)]$.

Similarly, by (7.6) and (7.7), we can rewrite the restriction of $p_{n}\left(t_{1}, 0,0, t_{4}\right)$ to $t_{1}=t_{4}=0$ as

$$
\lim _{\beta \rightarrow \infty} \rho^{n} m_{n}\left(\rho^{-1} x-\sigma ; \frac{1}{\beta-1}, \frac{1-c}{c(\beta-1)}\right)=2^{n / 2} h_{n}\left(2^{-1 / 2} x\right),
$$

where

$$
\rho=\frac{1-c}{c^{1 / 2}(\beta-1)^{1 / 2}}, \quad \sigma=-\frac{\beta c}{1-c} .
$$

This limit is equivalent to the limit given in [11, Sect. 2.4.4].

7.2 From Racah to Hermite along dual Hahn and Charlier

Next we will see the chart for the Racah manifold corresponding to the second graph in Fig. 2. Let $r_{n}$ again be the monic Racah polynomial given by (5.2). For $s_{1}, s_{2}, s_{3}, s_{4}>0$ and $s_{2}^{2} s_{4}<1$ put

$$
p_{n}(x)=p_{n}\left(x ; s_{1}, s_{2}, s_{3}, s_{4}\right):=\rho^{n} r_{n}\left(\rho^{-1} x-\sigma ; \alpha, \beta,-N-1, \delta\right),
$$

where

$$
\begin{aligned}
& \alpha=\frac{1+s_{1}}{s_{1} s_{2}}, \quad \beta=\frac{1+s_{1}}{s_{1} s_{2}^{2} s_{3} s_{4}}, \quad N=\frac{1}{s_{2}^{2} s_{4}}, \\
& \delta=\frac{1+s_{1}+s_{2} s_{4}\left(1+s_{1}+s_{1} s_{2}\right)}{s_{1} s_{2}^{2} s_{4}}, \\
& \rho=\frac{s_{1} s_{2}^{5 / 2} s_{4}}{2^{1 / 2}\left(1+s_{1}\right)}, \quad \sigma=-\frac{\left(1+s_{1}\right)\left(1+s_{3}-s_{2}^{2} s_{4}\right)+s_{1} s_{2}}{s_{1} s_{2}^{3} s_{4}} .
\end{aligned}
$$

Then the inequalities for $s_{1}, s_{2}, s_{3}, s_{4}$ are equivalent to (6.1). Furthermore, (6.3) holds with

$$
\begin{aligned}
B_{n}= & -2^{-1 / 2} s_{2}^{1 / 2}\left(2 n^{2}(n+1)^{2} s_{1}^{3} s_{2}^{6} s_{3}^{2} s_{4}^{3}\left(1+s_{1}\right)^{-1}\right. \\
& +4 n^{2}(n+1) s_{1}^{2} s_{2}^{4} s_{3} s_{4}^{2}\left(1+s_{2} s_{3} s_{4}\right)+n^{2} s_{1} s_{2}^{2} s_{4}\left(2+2 s_{1}-s_{3}-2 s_{1} s_{3}\right. \\
& -2 s_{1} s_{3}^{2}+5 s_{2} s_{3} s_{4}+5 s_{1} s_{2} s_{3} s_{4}+s_{2} s_{3}^{2} s_{4}+2 s_{1} s_{2} s_{3}^{2} s_{4}+4 s_{1} s_{2} s_{3}^{3} s_{4} \\
& \left.+3 s_{2}^{2} s_{3}^{2} s_{4}^{2}+3 s_{1} s_{2}^{2} s_{3}^{2} s_{4}^{2}-2 s_{1} s_{2}^{3} s_{3}^{2} s_{4}^{2}\right)-n\left(1+s_{1}+s_{2} s_{3} s_{4}\right.
\end{aligned}
$$




$$
\begin{aligned}
& \left.+s_{1} s_{2} s_{3} s_{4}+s_{1} s_{2}^{2} s_{3} s_{4}\right)\left(1+2 s_{1}+2 s_{1} s_{3}-s_{2} s_{4}-s_{1} s_{2} s_{4}\right. \\
& \left.-s_{2} s_{3} s_{4}-2 s_{1} s_{2} s_{3} s_{4}-4 s_{1} s_{2} s_{3}^{2} s_{4}-s_{2}^{2} s_{3} s_{4}^{2}-s_{1} s_{2}^{2} s_{3} s_{4}^{2}+2 s_{1} s_{2}^{2} s_{3} s_{4}^{2}\right) \\
& +\left(1+s_{1}\right)\left(1+s_{2} s_{3} s_{4}\right)\left(-s_{1} s_{3}-s_{2} s_{4}-s_{1} s_{2} s_{4}+s_{3}^{2} s_{4}+s_{1} s_{3}^{2} s_{4}\right. \\
& +2 s_{1} s_{2} s_{3}^{2} s_{4}-s_{2}^{2} s_{3} s_{4}^{2}-s_{1} s_{2}^{2} s_{3} s_{4}^{2} \\
& \left.\left.-2 s_{1} s_{2}^{3} s_{3} s_{4}^{2}\right)\right)\left(\left(1+s_{1}\right)\left(1+s_{2} s_{3} s_{4}\right)+2 n s_{1} s_{2}^{2} s_{3} s_{4}\right)^{-1} \\
& \times\left(\left(1+s_{1}\right)\left(1+s_{2} s_{3} s_{4}\right)+2(n+1) s_{1} s_{2}^{2} s_{3} s_{4}\right)^{-1}, \\
C_{n}= & n\left(1+s_{1}+n s_{1} s_{2}\right)\left(1+(1-n) s_{2}^{2} s_{4}\right)\left(1+s_{1}+(1-n) s_{1} s_{2}^{2} s_{4}\right) \\
& \times \frac{\left(1+s_{1}+n s_{1} s_{2}^{2} s_{3} s_{4}\right)\left(\left(1+s_{1}\right)\left(1+s_{2} s_{3} s_{4}\right)+s_{1} s_{3}+(n+1) s_{1} s_{2}^{2} s_{3} s_{4}\right)}{2\left(1+s_{1}\right)^{2}\left(\left(1+s_{1}\right)\left(1+s_{2} s_{3} s_{4}\right)+(2 n-1) s_{1} s_{2}^{2} s_{3} s_{4}\right)} \\
& \times \frac{\left(\left(1+s_{1}\right)\left(1+s_{2} s_{3} s_{4}\right)+n s_{1} s_{2}^{2} s_{3} s_{4}\right)\left(\left(1+s_{1}\right)\left(1+s_{3}+s_{2} s_{3} s_{4}\right)+(n+1) s_{1} s_{2}^{2} s_{3} s_{4}\right)}{\left(\left(1+s_{1}\right)\left(1+s_{2} s_{3} s_{4}\right)+2 n s_{1} s_{2}^{2} s_{3} s_{4}\right)^{2}\left(\left(1+s_{1}\right)\left(1+s_{2} s_{3} s_{4}\right)+(2 n+1) s_{1} s_{2}^{2} s_{3} s_{4}\right)} .
\end{aligned}
$$

Note that $B_{n}$ and $C_{n}$, as functions of $s_{1}, s_{2}, s_{3}, s_{4}>0$, can be uniquely extended to continuous functions of $s_{1}, s_{2}, s_{3}, s_{4} \geq 0$.

We now set one or more of the $s_{1}, s_{2}, s_{3}, s_{4}$ equal to zero and we proceed as in Sect. 7.1. We obtain:

dual Hahn,

$$
\begin{aligned}
& p_{n}\left(x ; s_{1}, s_{2}, 0, s_{4}\right)=\rho^{n} r_{n}^{\mathrm{DH}}\left(\rho^{-1} x-\sigma ; \gamma, \delta, N\right), \\
& \gamma=\frac{1+s_{1}}{s_{1} s_{2}}, \delta=\frac{1}{s_{1} s_{2}^{2} s_{4}}, N=\frac{1}{s_{2}^{2} s_{4}}, \\
& \rho=\frac{s_{1} s_{2}^{5 / 2} s_{4}}{2^{1 / 2}\left(1+s_{1}\right)}, \sigma=-\frac{\left(1+s_{1}\right)\left(1-s_{2}^{2} s_{4}\right)+s_{1} s_{2}}{s_{1} s_{2}^{2} s_{4}}
\end{aligned}
$$

\section{Meixner,}

$$
\begin{gathered}
p_{n}\left(x ; s_{1}, s_{2}, s_{3}, 0\right)=\rho_{0}^{n} p_{n}\left(\rho_{0}^{-1} x-\sigma_{0} ; s_{1}\left(1+s_{3}\right), \frac{s_{2}\left(1+s_{1}+s_{1} s_{3}\right)}{\left(1+s_{1}\right)\left(1+s_{3}\right)}, 0,0\right) \\
=\rho^{n} m_{n}\left(\rho^{-1} x-\sigma ; \beta, c\right), \\
\beta=\frac{1+s_{1}+s_{1} s_{2}}{s_{1} s_{2}}, c=\frac{s_{1}\left(1+s_{3}\right)}{1+s_{1}+s_{1} s_{3}}, \rho=\frac{s_{2}^{1 / 2}}{2^{1 / 2}\left(1+s_{1}\right)}, \\
\sigma=-\frac{1+s_{1}+s_{3}+s_{1} s_{2}+s_{1} s_{3}}{s_{2}}, \rho_{0}=\frac{\left(1+s_{3}\right)^{1 / 2}\left(1+s_{1}+s_{1} s_{3}\right)^{1 / 2}}{\left(1+s_{1}\right)^{1 / 2}}, \\
\sigma_{0} s_{2}^{1 / 2} s_{3}
\end{gathered}
$$




\section{Krawtchouk,}

$$
\begin{gathered}
p_{n}\left(x ; 0, s_{2}, s_{3}, s_{4}\right)=\rho_{0}^{n} p_{n}\left(\rho_{0}^{-1} x-\sigma_{0} ; \frac{s_{2}}{1+s_{3}+s_{2} s_{3} s_{4}}, 0, s_{4}\left(1+s_{3}+s_{2} s_{3} s_{4}\right)^{2}\right) \\
=\rho^{n} k_{n}\left(\rho^{-1} x-\sigma ; p, N\right), \\
p=\frac{s_{2} s_{4}\left(1+s_{3}+s_{2} s_{3} s_{4}\right)}{\left(1+s_{2} s_{4}\right)\left(1+s_{2} s_{3} s_{4}\right)}, N=\frac{1}{s_{2}^{2} s_{4}}, \rho=2^{-1 / 2} s_{2}^{1 / 2}\left(1+s_{2} s_{4}\right), \\
\sigma=-\frac{1+s_{3}-s_{2}^{2} s_{4}}{s_{2}\left(1+s_{2} s_{4}\right)}, \rho_{0}=\frac{\left(1+s_{3}+s_{2} s_{3} s_{4}\right)^{1 / 2}}{1+s_{2} s_{3} s_{4}}, \\
\sigma_{0}=-\frac{s_{2}^{1 / 2} s_{3} s_{4}\left(s_{2}+s_{3}\right)}{2^{1 / 2}\left(1+s_{3}+s_{2} s_{3} s_{4}\right)^{1 / 2}}
\end{gathered}
$$

\section{Charlier,}

$$
\begin{gathered}
p_{n}\left(x ; 0, s_{2}, s_{3}, 0\right)=\rho_{0}^{n} p_{n}\left(\rho_{0}^{-1} x ; 0, \frac{s_{2}}{1+s_{3}}, 0,0\right)=\rho^{n} c_{n}\left(\rho^{-1} x-\sigma ; a\right), \\
a=\frac{1+s_{3}}{s_{2}}, \rho=2^{-1 / 2} s_{2}^{1 / 2}, \sigma=-\frac{1+s_{3}}{s_{2}}, \rho_{0}=\left(1+s_{3}\right)^{1 / 2}
\end{gathered}
$$

\section{Hermite,}

$$
\begin{aligned}
p_{n}\left(x ; s_{1}, 0, s_{3}, s_{4}\right) & =p_{n}\left(x ; s_{1}, 0, s_{3}, 0\right)=p_{n}\left(x ; 0,0, s_{3}, 0\right) \\
& =\rho_{0}^{n} p_{n}\left(\rho_{0}^{-1} x ; 0,0, s_{3}, s_{4}\right) \\
& =\rho_{0}^{n} p_{n}\left(\rho_{0}^{-1} x ; 0,0, s_{3}, 0\right)=\rho^{n} p_{n}\left(\rho^{-1} x ; s_{1}, 0,0, s_{4}\right) \\
& =\rho^{n} p_{n}\left(\rho^{-1} x ; 0,0,0, s_{4}\right)=\rho^{n} h_{n}\left(\rho^{-1} x\right), \\
\rho=\frac{\left(1+s_{3}\right)^{1 / 2}}{\left(1+s_{1}+s_{1} s_{3}\right)^{1 / 2}} & \rho_{0}=\frac{\left(1+s_{1}+s_{1} s_{3}\right)^{1 / 2}}{\left(1+s_{1}\right)^{1 / 2}} .
\end{aligned}
$$

The various parameter restrictions of the polynomial (7.8) are summarized in Fig. 5. Even more than in Fig. 4, several boxes essentially coincide.

\subsection{From Racah to Hermite along dual Hahn and Laguerre}

Here we will see the chart for the Racah manifold corresponding to the third graph in Fig. 2. Let $r_{n}$ again be the monic Racah polynomial given by (5.2). For $u_{1}, u_{2}$, $u_{3}, u_{4}>0, u_{2}^{2} u_{3} u_{4}<1$ and $u_{2} u_{3}\left(u_{1}-u_{2}\right)<1$ put

$$
p_{n}(x)=p_{n}\left(x ; u_{1}, u_{2}, u_{3}, u_{4}\right):=\rho^{n} r_{n}\left(\rho^{-1} x-\sigma ; \alpha, \beta,-N-1, \delta\right),
$$




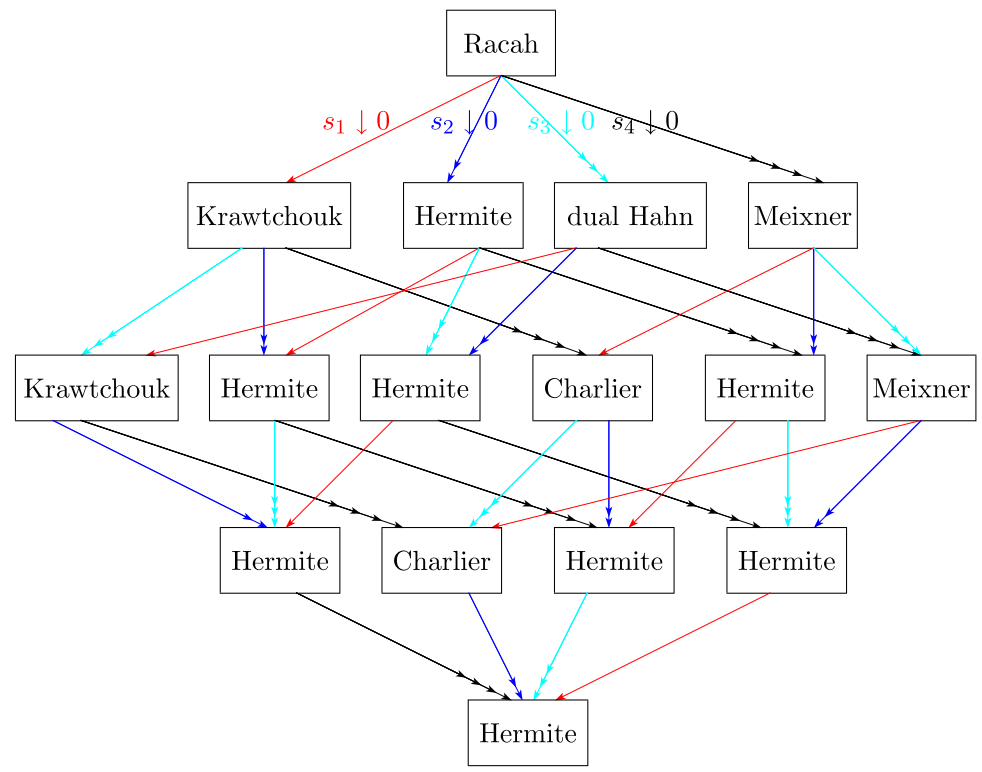

Fig. 5 The various parameter restrictions in the second chart for the Racah manifold

where

$$
\begin{aligned}
& \alpha=\frac{1+u_{1}}{u_{2}}, \quad \beta=\frac{1}{u_{1} u_{2}^{2} u_{3}^{2} u_{4}}, \quad N=\frac{1}{u_{2}^{2} u_{3} u_{4}}, \\
& \delta=\frac{1+u_{4}+u_{2} u_{3} u_{4}+u_{2}^{2} u_{3} u_{4}}{u_{2}^{2} u_{3} u_{4}}, \quad \rho=2^{-1 / 2} u_{2}^{5 / 2} u_{3} u_{4}, \\
& \sigma=-\frac{\left(1+u_{1}\right)\left(1+u_{1} u_{3}+u_{1} u_{3} u_{4}\right)}{u_{2}^{3} u_{3} u_{4}} .
\end{aligned}
$$

Then the inequalities for $t_{1}, t_{2}, t_{3}, t_{4}$ are equivalent to (6.1). Furthermore, (6.3) holds with

$$
\begin{aligned}
B_{n}= & -2^{-1 / 2} u_{2}^{1 / 2}\left(2 n^{2}(n+1)^{2} u_{1}^{2} u_{2}^{6} u_{3}^{5} u_{4}^{3}+4 n^{2}(n+1) u_{1} u_{2}^{4} u_{3}^{3} u_{4}^{2}\right. \\
& \times\left(1+u_{1} u_{2} u_{3}^{2} u_{4}+u_{1}^{2} u_{2} u_{3}^{2} u_{4}\right) \\
& +n^{2} u_{2}^{2} u_{3} u_{4}\left(2-2 u_{1} u_{3}-2 u_{1}^{2} u_{3}^{2}-u_{1} u_{3} u_{4}-2 u_{1}^{2} u_{3}^{2} u_{4}+5 u_{1} u_{2} u_{3}^{2} u_{4}\right. \\
& +6 u_{1}^{2} u_{2} u_{3}^{3} u_{4}+2 u_{1}^{2} u_{2} u_{3}^{3} u_{4}+4 u_{1}^{3} u_{2} u_{3}^{3} u_{4}-4 u_{1}^{2} u_{2}^{2} u_{3}^{3} u_{4}+4 u_{1}^{3} u_{2} u_{3}^{4} u_{4} \\
& +4 u_{1}^{4} u_{2} u_{3}^{4} u_{4}+u_{1}^{2} u_{2} u_{3}^{3} u_{4}^{2}+u_{1}^{3} u_{2} u_{3}^{3} u_{4}^{2}+4 u_{1}^{3} u_{2} u_{3}^{4} u_{4}^{2}+4 u_{1}^{4} u_{2} u_{3}^{4} u_{4}^{2} \\
& \left.+3 u_{1}^{2} u_{2}^{2} u_{3}^{4} u_{4}^{2}+5 u_{1}^{3} u_{2}^{2} u_{3}^{4} u_{4}^{2}+2 u_{1}^{4} u_{2}^{2} u_{3}^{4} u_{4}^{2}+2 u_{1}^{2} u_{2}^{3} u_{3}^{4} u_{4}^{2}+2 u_{1}^{3} u_{2}^{3} u_{3}^{4} u_{4}^{2}\right) \\
& +n\left(1+u_{1} u_{2} u_{3}^{2} u_{4}+u_{1}^{2} u_{2} u_{3}^{2} u_{4}+u_{1} u_{2}^{2} u_{3}^{2} u_{4}\right)\left(-2-2 u_{1} u_{3}-u_{4}\right.
\end{aligned}
$$




$$
\begin{aligned}
& -2 u_{1} u_{3} u_{4}+u_{2} u_{3} u_{4}+2 u_{1} u_{2} u_{3} u_{4}+2 u_{1} u_{2} u_{3}^{2} u_{4}+4 u_{1}^{2} u_{2} u_{3}^{2} u_{4} \\
& -4 u_{1} u_{2}^{2} u_{3}^{2} u_{4}+4 u_{1}^{2} u_{2} u_{3}^{3} u_{4}+4 u_{1}^{3} u_{2} u_{3}^{3} u_{4}+u_{1} u_{2} u_{3}^{2} u_{4}^{2}+u_{1}^{2} u_{2} u_{3}^{2} u_{4}^{2} \\
& +4 u_{1}^{2} u_{2} u_{3}^{3} u_{4}^{2}+4 u_{1}^{3} u_{2} u_{3}^{3} u_{4}^{2}+u_{1} u_{2}^{2} u_{3}^{3} u_{4}^{2}+u_{1}^{2} u_{2}^{2} u_{3}^{3} u_{4}^{2}+2 u_{1} u_{2}^{3} u_{3}^{3} u_{4}^{2} \\
& \left.+2 u_{1}^{2} u_{2}^{3} u_{3}^{3} u_{4}^{2}\right)+\left(1+u_{1} u_{2} u_{3}^{2} u_{4}+u_{1}^{2} u_{2} u_{3}^{2} u_{4}\right)\left(-1-u_{1} u_{3}-u_{1} u_{3} u_{4}\right. \\
& +u_{1}^{2} u_{3}^{2} u_{4}+u_{1}^{3} u_{3}^{2} u_{4}-u_{1} u_{2} u_{3}^{2} u_{4}-2 u_{1} u_{2}^{2} u_{3}^{2} u_{4}+u_{1}^{2} u_{3}^{3} u_{4}+2 u_{1}^{3} u_{3}^{3} u_{4} \\
& +u_{1}^{4} u_{3}^{3} u_{4}+2 u_{1}^{2} u_{2} u_{3}^{3} u_{4}+2 u_{1}^{3} u_{2} u_{3}^{3} u_{4}+u_{1}^{2} u_{3}^{3} u_{4}^{2}+2 u_{1}^{3} u_{3}^{3} u_{4}^{2}+u_{1}^{4} u_{3}^{3} u_{4}^{2} \\
& \left.\left.+2 u_{1}^{2} u_{2} u_{3}^{3} u_{4}^{2}+2 u_{1}^{3} u_{2} u_{3}^{3} u_{4}^{2}\right)\right)\left(1+u_{1} u_{2} u_{3}^{2} u_{4}\left(1+u_{1}\right)+2 n u_{1} u_{2}^{2} u_{3}^{2} u_{4}\right)^{-1} \\
& \times\left(1+u_{1} u_{2} u_{3}^{2} u_{4}\left(1+u_{1}\right)+2(n+1) u_{1} u_{2}^{2} u_{3}^{2} u_{4}\right)^{-1} \\
C_{n} & \frac{1}{2} \\
& n\left(1+u_{1}+n u_{2}\right)\left(1+(1-n) u_{2}^{2} u_{3} u_{4}\right) \\
& \times\left(1+u_{4}-u_{1} u_{2} u_{3} u_{4}+(1-n) u_{2}^{2} u_{3} u_{4}\right) \\
& \times \frac{\left(1+u_{1} u_{2} u_{3}^{2} u_{4}\left(1+u_{1}\right)+n u_{1} u_{2}^{2} u_{3}^{2} u_{4}\right)\left(1+u_{1} u_{3}\left(1+u_{4}+u_{2} u_{3} u_{4}\right)+(n+1) u_{1} u_{2}^{2} u_{3}^{2} u_{4}\right)}{\left(1+u_{1} u_{2} u_{3}^{2} u_{4}\left(1+u_{1}\right)+(2 n-1) u_{1} u_{2}^{2} u_{3}^{2} u_{4}\right)\left(1+u_{1} u_{2} u_{3}^{2} u_{4}\left(1+u_{1}\right)+2 n u_{1} u_{2}^{2} u_{3}^{2} u_{4}\right)^{2}} \\
& \times \frac{\left(1+n u_{1} u_{2}^{2} u_{3}^{2} u_{4}\right)\left(1+u_{1} u_{3}\left(1+u_{2} u_{3} u_{4}+u_{1} u_{2} u_{3} u_{4}\right)+(n+1) u_{1} u_{2}^{2} u_{3}^{2} u_{4}\right)}{1+u_{1} u_{2} u_{3}^{2} u_{4}\left(1+u_{1}\right)+(2 n+1) u_{1} u_{2}^{2} u_{3}^{2} u_{4}}
\end{aligned}
$$

Note that $B_{n}$ and $C_{n}$, as functions of $u_{1}, u_{2}, u_{3}, u_{4}>0$, can be uniquely extended to continuous functions of $u_{1}, u_{2}, u_{3}, u_{4} \geq 0$.

We now set one or more of the $u_{1}, u_{2}, u_{3}, u_{4}$ equal to zero and we proceed as in Sect. 7.1. We obtain:

dual Hahn,

$$
\begin{aligned}
& p_{n}\left(x ; 0, u_{2}, u_{3}, u_{4}\right)=\rho^{n} r_{n}^{\mathrm{DH}}\left(\rho^{-1} x-\sigma ; \gamma, \delta, N\right), \\
& \quad \gamma=\frac{1}{u_{2}}, \delta=\frac{1}{u_{2}^{2} u_{3}}, N=\frac{1}{u_{2}^{2} u_{3} u_{4}}, \rho=2^{-1 / 2} u_{2}^{5 / 2} u_{3} u_{4}, \sigma=-\frac{1}{u_{2}^{2} u_{3} u_{4}} ;
\end{aligned}
$$

\section{Meixner,}

$$
\begin{aligned}
& p_{n}\left(x ; u_{1}, u_{2}, 0, u_{4}\right)=\rho_{0}^{n} p_{n}\left(\rho_{0}^{-1} x ; 0, \frac{u_{2}}{1+u_{1}}, 0, u_{4}\right)=\rho^{n} m_{n}\left(\rho^{-1} x-\sigma ; \beta, c\right), \\
& \beta=\frac{1+u_{1}+u_{2}}{u_{2}}, c=\frac{1}{1+u_{4}}, \rho=2^{-1 / 2} u_{2}^{1 / 2} u_{4}, \rho_{0}=\left(1+u_{1}\right)^{1 / 2}, \\
& \sigma=-\frac{1+u_{1}}{u_{2} u_{4}}
\end{aligned}
$$




\section{Laguerre,}

$$
\begin{aligned}
p_{n}\left(x ; u_{1}, u_{2}, u_{3}, 0\right) & =\rho_{1}^{n} p_{n}\left(\rho_{1}^{-1} x ; u_{1}, u_{2}, 0,0\right)=\rho_{0}^{n} p_{n}\left(\rho_{0}^{-1} x ; 0, \frac{u_{2}}{1+u_{1}}, u_{3}, 0\right) \\
& =\rho_{0}^{n} p_{n}\left(\rho_{0}^{-1} x ; 0, \frac{u_{2}}{1+u_{1}}, 0,0\right)=\rho^{n} \ell_{n}^{(\alpha)}\left(\rho^{-1} x-\sigma\right), \\
\alpha=\frac{1+u_{1}}{u_{2}}, \rho= & 2^{-1 / 2} u_{2}^{1 / 2}\left(1+u_{1} u_{3}\right), \rho_{0}=\left(1+u_{1}\right)^{1 / 2}\left(1+u_{1} u_{3}\right), \\
\rho_{1}=1+u_{1} u_{3}, \sigma & =-\frac{1+u_{1}}{u_{2}} ;
\end{aligned}
$$

\section{Hermite,}

$$
\begin{gathered}
p_{n}\left(x ; u_{1}, 0, u_{3}, u_{4}\right)=\rho_{0}^{n} p_{n}\left(\rho_{0}^{-1} x ; 0,0, u_{3}, u_{4}\right)=\rho_{0}^{n} p_{n}\left(\rho_{0}^{-1} x ; 0,0,0, u_{4}\right) \\
=\rho_{1}^{n} p_{n}\left(\rho_{1}^{-1} x ; u_{1}, 0,0, u_{4}\right)=\rho_{2}^{n} p_{n}\left(\rho_{2}^{-1} x ; u_{1}, 0, u_{3}, 0\right) \\
=\rho_{3}^{n} p_{n}\left(\rho_{3}^{-1} x ; u_{1}, 0,0,0\right) \\
=\rho^{n} p_{n}\left(\rho^{-1} x ; 0,0, u_{3}, 0\right)=\rho^{n} p_{n}\left(\rho^{-1} x ; 0,0,0,0\right) \\
=\rho^{n} h_{n}\left(\rho^{-1} x\right), \\
\rho_{0}=\left(1+u_{1}\right)^{1 / 2}\left(1+u_{1} u_{3}\right)^{1 / 2}\left(1+u_{4}\right)^{1 / 2}\left(1+u_{1} u_{3}\left(1+u_{4}\right)\right)^{1 / 2}, \\
\rho_{1}=\left(1+u_{1}\right)^{1 / 2}\left(1+u_{1} u_{3}\right)^{1 / 2}\left(1+u_{1} u_{3}\left(1+u_{4}\right)\right)^{1 / 2}, \\
\rho_{2}=\frac{\left(1+u_{4}\right)^{1 / 2}\left(1+u_{1} u_{3}\left(1+u_{4}\right)\right)^{1 / 2}}{\left(1+u_{1} u_{3}\right)^{1 / 2}}, \\
\rho_{3}=\left(1+u_{4}\right)^{1 / 2}\left(1+u_{1} u_{3}\right)^{1 / 2}\left(1+u_{1} u_{3}\left(1+u_{4}\right)\right)^{1 / 2} .
\end{gathered}
$$

The various parameter restrictions of the polynomial (7.10) are summarized in Fig. 6. Many boxes essentially coincide.

7.4 Transformations between the local charts

By (7.2), (7.9) and (7.11), we can compute the transformations between the local coordinates $\left(t_{1}, t_{2}, t_{3}, t_{4}\right),\left(s_{1}, s_{2}, s_{3}, s_{4}\right)$ and $\left(u_{1}, u_{2}, u_{3}, u_{4}\right)$.

first chart $\leftrightarrow$ second chart

$$
\begin{gathered}
t_{1}=\frac{s_{1} s_{2}}{1+s_{1}}, \quad t_{2}=s_{2} s_{3} s_{4}, \quad t_{3}=\frac{1+s_{1}}{s_{2}\left(1+s_{1}+s_{1} s_{2}^{2} s_{4}\right)}, \quad t_{4}=\frac{s_{2}}{s_{3}} ; \\
s_{1}=\frac{t_{1} t_{3}}{1-t_{1} t_{3}-t_{1} t_{2} t_{3} t_{4}}, s_{2}=\frac{1-t_{1} t_{2} t_{3} t_{4}}{t_{3}}, s_{3}=\frac{1-t_{1} t_{2} t_{3} t_{4}}{t_{3} t_{4}},
\end{gathered}
$$




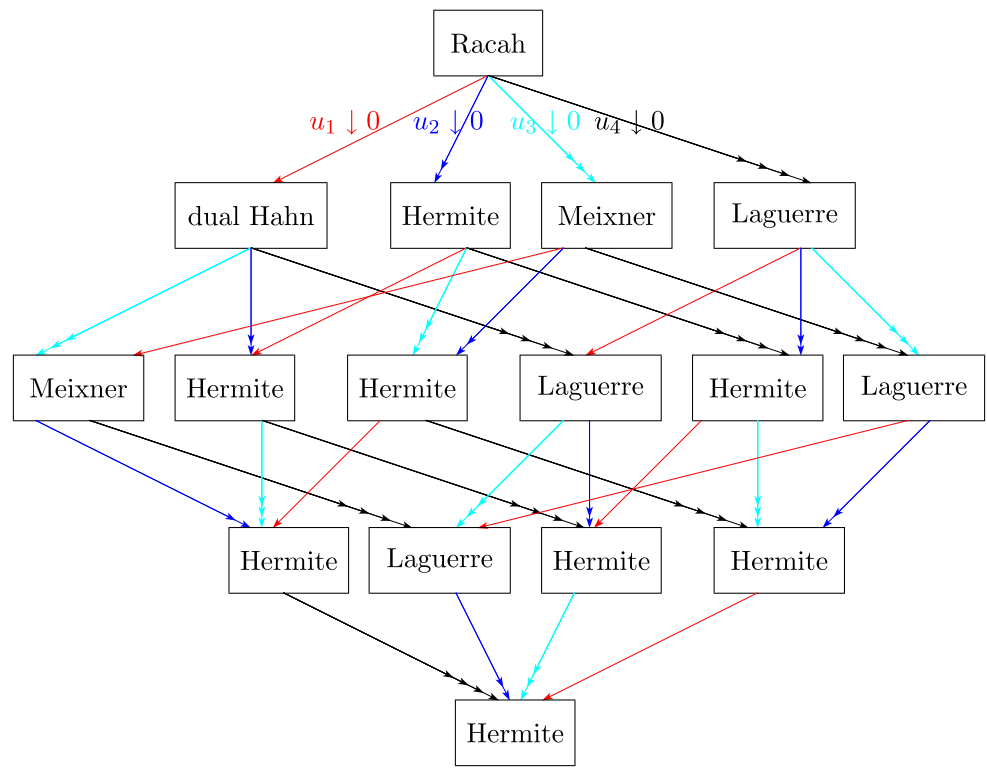

Fig. 6 The various parameter restrictions in the third chart for the Racah manifold

$$
s_{4}=\frac{t_{2} t_{3}^{2} t_{4}}{\left(1-t_{1} t_{2} t_{3} t_{4}\right)^{2}} .
$$

This is a homeomorphism between

$$
\left\{\left(t_{1}, t_{2}, t_{3}, t_{4}\right) \mid t_{1} \geq 0, t_{2} \geq 0, t_{3}>0, t_{4}>0, t_{1} t_{3}\left(1+t_{2} t_{4}\right)<1, t_{2} t_{4}<1\right\}
$$

and

$$
\left\{\left(s_{1}, s_{2}, s_{3}, s_{4}\right) \mid s_{1} \geq 0, s_{2}>0, s_{3}>0, s_{4} \geq 0, s_{2}^{2} s_{4}<1\right\} .
$$

It also identifies the Krawtchouk box $\left\{t_{1}=0\right\}$, Meixner box $\left\{t_{2}=0\right\}$ and Charlier box $\left\{t_{1}=t_{2}=0\right\}$ in Fig. 4 with the corresponding boxes $\left\{s_{1}=0\right\},\left\{s_{4}=0\right\}$ and $\left\{s_{1}=s_{4}=\right.$ $0\}$ in Fig. 5.

second chart $\leftrightarrow$ third chart

$$
\begin{aligned}
& s_{1}=\frac{1}{u_{4}\left(1-u_{1} u_{2} u_{3}\right)}, \quad s_{2}=\frac{u_{2}\left(1+u_{4}\left(1-u_{1} u_{2} u_{3}\right)\right)}{1+u_{1}} \\
& s_{3}=u_{1} u_{3}\left(1+u_{4}\left(1-u_{1} u_{2} u_{3}\right)\right), \quad s_{4}=\frac{\left(1+u_{1}\right)^{2} u_{3} u_{4}}{\left(1+u_{4}\left(1-u_{1} u_{2} u_{3}\right)\right)^{2}} \\
& u_{1}=\frac{-2 s_{1}^{2} s_{3}+s_{4}\left(1+s_{1}\right)\left(1+2 s_{1}+s_{1}^{2}-s_{1}^{2} s_{2} s_{3}\right)-\left(1+s_{1}\right) S^{1 / 2}}{2 s_{1}^{2} s_{3}\left(1+s_{2} s_{4}+s_{1} s_{2} s_{4}\right)} \\
& u_{2}=\frac{s_{1} s_{2}^{2} s_{4}}{1+s_{2} s_{4}+s_{1} s_{2} s_{4}}+s_{2} \frac{s_{4}\left(1+2 s_{1}+s_{1}^{2}-s_{1}^{2} s_{2} s_{3}\right)-S^{1 / 2}}{2 s_{1} s_{3}\left(1+s_{2} s_{4}+s_{1} s_{2} s_{4}\right)}
\end{aligned}
$$




$$
\begin{aligned}
& u_{3}=\frac{-2 s_{1}^{2} s_{3}+s_{4}\left(1+s_{1}\right)\left(1+2 s_{1}+s_{1}^{2}-s_{1}^{2} s_{2} s_{3}\right)+\left(1+s_{1}\right) S^{1 / 2}}{2 s_{1}\left(1+s_{1}\right)}, \\
& u_{4}=\frac{1}{s_{1}}+s_{2} \frac{s_{4}\left(1+2 s_{1}+s_{1}^{2}-s_{1}^{2} s_{2} s_{3}\right)-S^{1 / 2}}{2 s_{1}\left(1+s_{1}\right)}
\end{aligned}
$$

where

$$
S:=s_{4}^{2}\left(1+2 s_{1}+s_{1}^{2}-s_{1}^{2} s_{2} s_{3}\right)^{2}-4 s_{1}^{2} s_{3} s_{4}\left(1+s_{1}\right) .
$$

This is a homeomorphism between

$$
\begin{aligned}
\left\{\left(s_{1}, s_{2}, s_{3}, s_{4}\right) \mid\right. & s_{1}>0, s_{2} \geq 0, s_{3}>0, s_{4}>0, s_{2}^{2} s_{4}<1, S \geq 0, \\
& \left.-2 s_{1}^{2} s_{3}+s_{4}\left(1+s_{1}\right)\left(1+2 s_{1}+s_{1}^{2}-s_{1}^{2} s_{2} s_{3}\right) \geq 0\right\}
\end{aligned}
$$

and

$$
\begin{gathered}
\left\{u_{1}, u_{2}, u_{3}, u_{4}>0 \mid u_{1}>0, u_{2} \geq 0, u_{3}>0, u_{4}>0, u_{2}^{2} u_{3} u_{4}<1,\right. \\
\left.u_{2} u_{3}\left(u_{1}-u_{2}\right)<1, u_{1}\left(1+u_{2} u_{3}\right) \leq 1\right\} .
\end{gathered}
$$

It also identifies the Hermite box $\left\{s_{2}=0\right\}$ in Fig. 5 with the Hermite box $\left\{u_{2}=0\right\}$ in Fig. 6.

first chart $\leftrightarrow$ third chart

$$
\begin{gathered}
t_{1}=\frac{u_{2}}{1+u_{1}}, \quad t_{2}=u_{1} u_{2} u_{3}^{2} u_{4}\left(1+u_{1}\right), \\
t_{3}=\frac{1+u_{1}}{u_{2}\left(1+u_{4}\left(1-u_{2} u_{3}\left(u_{1}-u_{2}\right)\right)\right)}, \quad t_{4}=\frac{u_{2}}{u_{1} u_{3}\left(1+u_{1}\right)} ; \\
u_{1}=\frac{t_{2} t_{3} t_{4}\left(t_{4}-t_{1}^{2}\right)-2 t_{1}^{2}\left(1-t_{1} t_{3}\left(1+t_{2} t_{4}\right)\right)-t_{4} T^{1 / 2}}{2 t_{1}^{2}\left(1-t_{1} t_{3}\left(1+t_{2} t_{4}\right)+t_{2} t_{3} t_{4}\right)}, \\
u_{2}=\frac{t_{2} t_{3} t_{4}\left(t_{1}^{2}+t_{4}\right)-t_{4} T^{1 / 2}}{2 t_{1}\left(1-t_{1} t_{3}\left(1+t_{2} t_{4}\right)+t_{2} t_{3} t_{4}\right)}, \\
u_{3}=\frac{t_{2} t_{3} t_{4}\left(t_{4}-t_{1}^{2}\right)-2 t_{1}^{2}\left(1-t_{1} t_{3}\left(1+t_{2} t_{4}\right)\right)+t_{4} T^{1 / 2}}{2 t_{1}\left(1-t_{1} t_{3}\left(1+t_{2} t_{4}\right)\right)}, \\
u_{4}=\frac{2\left(1-t_{1} t_{3}\left(1+t_{2} t_{4}\right)\right)+t_{2} t_{3}\left(t_{4}-t_{1}^{2}\right)-T^{1 / 2}}{2 t_{1} t_{3}}
\end{gathered}
$$

where

$$
T:=t_{2}^{2} t_{3}^{2}\left(t_{4}-t_{1}^{2}\right)^{2}-4 t_{1}^{2} t_{2} t_{3}\left(1-t_{1} t_{3}\left(1+t_{2} t_{4}\right)\right) .
$$

This is a homeomorphism between

$$
\begin{array}{r}
\left\{\left(t_{1}, t_{2}, t_{3}, t_{4}\right) \mid t_{1}>0, t_{2}>0, t_{3}>0, t_{4}>0, t_{2} t_{4}<1, T \geq 0,\right. \\
\left.t_{2} t_{3} t_{4}\left(t_{4}-t_{1}^{2}\right) \geq 2 t_{1}^{2}\left(1-t_{1} t_{3}\left(1+t_{2} t_{4}\right)\right)>0\right\}
\end{array}
$$


and

$$
\begin{gathered}
\left\{u_{1}, u_{2}, u_{3}, u_{4}>0 \mid u_{1}>0, u_{2}>0, u_{3}>0, u_{4}>0, u_{2}^{2} u_{3} u_{4}<1,\right. \\
\left.u_{2} u_{3}\left(u_{1}-u_{2}\right)<1, u_{1}\left(1+u_{2} u_{3}\right) \leq 1\right\} .
\end{gathered}
$$

\section{The two Wilson manifolds}

In this section, I will present in detail the two charts for the two Wilson manifolds, as introduced in Sect. 6, and corresponding to the two graphs in Fig. 3.

\subsection{From Wilson to Hermite along Continuous Hahn}

Here we will see the chart for the Wilson manifold corresponding to the first graph in Fig. 3. The monic Wilson polynomial $w_{n}$ is given by (5.4). For $a_{1}, a_{2}, a_{3}, a_{4}>0$ put

$$
p_{n}(x)=p_{n}\left(x ; a_{1}, a_{2}, a_{3}, a_{4}\right):=\rho^{n} w_{n}\left(\rho^{-1} x-\sigma ; a, b, c, d\right),
$$

where

$$
\begin{aligned}
& a=a_{1}^{-1}-\frac{1-a_{1}^{1 / 2} a_{2} a_{4}}{2 a_{1}^{3 / 2} a_{2}^{2} a_{3} a_{4}} i, \quad b=a_{1}^{-1} a_{2}^{-1}-\frac{1+a_{1}^{1 / 2} a_{2} a_{4}}{2 a_{1}^{3 / 2} a_{2}^{2} a_{3} a_{4}} i, \\
& c=a_{1}^{-1}+\frac{1-a_{1}^{1 / 2} a_{2} a_{4}}{2 a_{1}^{3 / 2} a_{2}^{2} a_{3} a_{4}} i, \quad d=a_{1}^{-1} a_{2}^{-1}+\frac{1+a_{1}^{1 / 2} a_{2} a_{4}}{2 a_{1}^{3 / 2} a_{2}^{2} a_{3} a_{4}} i, \\
& \rho=2^{3 / 2} a_{1}^{2} a_{2}^{2} a_{3}^{2} a_{4}, \quad \sigma=-\frac{1}{4 a_{1}^{3} a_{2}^{4} a_{3}^{2} a_{4}^{2}}+\frac{1-a_{2}}{2 a_{1}^{5 / 2} a_{2}^{3}\left(1+a_{2}-a_{1} a_{2}\right) a_{3}^{2} a_{4}} .
\end{aligned}
$$

Then (6.3) holds with

$$
\begin{aligned}
B_{n}= & 2^{-1 / 2}\left(2 n^{4} a_{1}^{4} a_{2}^{4} a_{3}^{2} a_{4}\left(1+a_{2}-a_{1} a_{2}\right)\right. \\
& +4 n^{3} a_{1}^{3} a_{2}^{3} a_{3}^{2} a_{4}\left(2+2 a_{2}-a_{1} a_{2}\right)\left(1+a_{2}-a_{1} a_{2}\right) \\
& +n^{2} a_{1}^{3 / 2} a_{2}\left(2-2 a_{2}+a_{1}^{1 / 2} a_{2} a_{4}\left(1+a_{2}-a_{1} a_{2}\right)\right. \\
& +a_{1}^{1 / 2} a_{2} a_{3}^{2} a_{4}\left(10+34 a_{2}-20 a_{1} a_{2}+34 a_{2}^{2}-44 a_{1} a_{2}^{2}+12 a_{1}^{2} a_{2}^{2}+10 a_{2}^{3}\right. \\
& \left.\left.-20 a_{1} a_{2}^{3}+12 a_{1}^{2} a_{2}^{3}-2 a_{1}^{3} a_{2}^{3}\right)\right)+n a_{1}^{1 / 2}\left(2+2 a_{2}-a_{1} a_{2}\right)\left(2-2 a_{2}\right. \\
& +a_{1}^{1 / 2} a_{2} a_{4}\left(1+a_{2}-a_{1} a_{2}\right)+2 a_{1}^{1 / 2} a_{2} a_{3}^{2} a_{4}\left(1+5 a_{2}-2 a_{1} a_{2}+5 a_{2}^{2}\right. \\
& \left.\left.-6 a_{1} a_{2}^{2}+a_{1}^{2} a_{2}^{2}+a_{2}^{3}-2 a_{1} a_{2}^{3}+a_{1}^{2} a_{2}^{3}\right)\right) \\
& +\left(1+a_{2}-a_{1} a_{2}\right)\left(2 a_{1}^{1 / 2}-2 a_{1}^{1 / 2} a_{2}+a_{4}+2 a_{2} a_{4}-a_{1} a_{2} a_{4}+a_{2}^{2} a_{4}\right. \\
& \left.\left.-a_{1} a_{2}^{2} a_{4}+4 a_{2} a_{3}^{2} a_{4}\left(1+2 a_{2}-a_{1} a_{2}+a_{2}^{2}-a_{1} a_{2}^{2}\right)\right)\right) \\
& \times\left(1+a_{2}-a_{1} a_{2}\right)^{-1}\left(1+a_{2}+(n-1) a_{1} a_{2}\right)^{-1}\left(1+a_{2}+n a_{1} a_{2}\right)^{-1},
\end{aligned}
$$




$$
\begin{aligned}
C_{n}= & \frac{1}{2} n\left(1+a_{3}^{2}\left(1+a_{2}+(n-1) a_{1} a_{2}\right)^{2}\right) \\
& \times\left(1+a_{1} a_{2}^{2} a_{3}^{2} a_{4}^{2}\left(1+a_{2}+(n-1) a_{1} a_{2}\right)^{2}\right) \\
& \times \frac{\left(2-a_{1}+n a_{1}\right)\left(2+2 a_{2}+(n-2) a_{1} a_{2}\right)\left(2+(n-1) a_{1} a_{2}\right)}{\left(1+a_{2}+\left(n-\frac{1}{2}\right) a_{1} a_{2}\right)\left(1+a_{2}+(n-1) a_{1} a_{2}\right)^{2}\left(1+a_{2}+\left(n-\frac{3}{2}\right) a_{1} a_{2}\right)} .
\end{aligned}
$$

Note that $B_{n}$ and $C_{n}$, as functions of $a_{1}, a_{2}, a_{3}, a_{4}>0$, can be uniquely extended to continuous functions of $a_{1}, a_{2}, a_{3}, a_{4} \geq 0$.

We now set one or more of the $a_{1}, a_{2}, a_{3}, a_{4}$ equal to zero in (8.2) and (8.3) and we proceed as in Sect. 7.1. We obtain:

\section{Continuous Hahn,}

$$
\begin{aligned}
& p_{n}\left(x ; a_{1}, a_{2}, a_{3}, 0\right)=\rho^{n} p_{n}^{\mathrm{CH}}\left(\rho^{-1} x-\sigma ; a, b, c, d\right), \\
& a=\frac{i+2 a_{2} a_{3}}{2 a_{1} a_{2} a_{3}}, b=\frac{-i+2 a_{3}}{2 a_{1} a_{2} a_{3}}, c=\frac{-i+2 a_{2} a_{3}}{2 a_{1} a_{2} a_{3}}, d=\frac{i+2 a_{3}}{2 a_{1} a_{2} a_{3}}, \\
& \rho=2^{3 / 2} a_{1}^{1 / 2} a_{3}, \sigma=\frac{1-a_{2}}{2 a_{1} a_{2} a_{3}\left(1+a_{2}-a_{1} a_{2}\right)} ;
\end{aligned}
$$

Jacobi,

$$
\begin{gathered}
p_{n}\left(x ; a_{1}, a_{2}, 0, a_{4}\right)=p_{n}\left(x-\sigma_{0} ; a_{1}, a_{2}, 0,0\right)=\rho^{n} p_{n}^{(\alpha, \beta)}\left(\rho^{-1} x-\sigma\right), \\
\alpha=2 a_{1}^{-1}-1, \beta=2 a_{1}^{-1} a_{2}^{-1}-1, \rho=-2^{1 / 2} a_{1}^{-1 / 2} a_{2}^{-1}, \\
\sigma=-\frac{1-a_{2}}{1+a_{2}-a_{1} a_{2}}-\frac{1}{2} a_{1}^{1 / 2} a_{2} a_{4}, \sigma_{0}=2^{-1 / 2} a_{4} ;
\end{gathered}
$$

Meixner-Pollaczek,

$$
\begin{aligned}
& p_{n}\left(x ; a_{1}, 0, a_{3}, a_{4}\right)=p_{n}\left(x-\sigma_{0} ; a_{1}, 0, a_{3}, 0\right)=\rho^{n} p_{n}^{(\lambda)}\left(\rho^{-1} x-\sigma\right), \\
& \lambda=a_{1}{ }^{-1}, \phi=\arctan \left(a_{3}\right), \rho=-2^{3 / 2} a_{1}^{1 / 2} a_{3}, \\
& \sigma=\frac{2-a_{1}}{2 a_{1} a_{3}}-\frac{1}{4} a_{1}^{-1 / 2} a_{3}^{-1} a_{4}, \sigma_{0}=2^{-1 / 2} a_{4} ;
\end{aligned}
$$

\section{Laguerre,}

$$
\begin{aligned}
& p_{n}\left(x ; a_{1}, 0,0, a_{4}\right)=p_{n}\left(x-\sigma_{0} ; a_{1}, 0,0,0\right)=\rho^{n} \ell_{n}^{(\alpha)}\left(\rho^{-1} x-\sigma\right), \\
& \alpha=2 a_{1}^{-1}-1, \rho=2^{1 / 2} a_{1}^{1 / 2}, \sigma=1-2 a_{1}^{-1}+\frac{1}{2} a_{1}^{-1 / 2} a_{4}, \sigma_{0}=2^{-1 / 2} a_{4} ;
\end{aligned}
$$




\section{Hermite,}

$$
\begin{aligned}
& p_{n}\left(x ; 0, a_{2}, a_{3}, a_{4}\right)=p_{n}\left(x-\sigma_{0} ; 0, a_{2}, a_{3}, 0\right)=\rho_{1}^{n} p_{n}\left(\rho_{1}^{-1} x-\sigma_{1} ; 0, a_{2}, 0, a_{4}\right) \\
& =\rho_{2}^{n} p_{n}\left(\rho_{2}^{-1} x-\sigma_{2} ; 0,0, a_{3}, a_{4}\right) \\
& =\rho_{1}^{n} p_{n}\left(\rho_{1}^{-1} x-\sigma_{3} ; 0, a_{2}, 0,0\right) \\
& =\rho^{n} p_{n}\left(\rho^{-1} x-\sigma_{4} ; 0,0, a_{3}, 0\right)=\rho_{2}^{n} p_{n}\left(\rho_{2}^{-1} x-\sigma_{5} ; 0,0,0, a_{4}\right) \\
& =\left(2^{-3 / 2} \rho\right)^{n} p_{n}\left(2^{3 / 2}\left(\rho^{-1} x-\sigma\right) ; 0,0,0,0\right)=\rho^{n} h_{n}\left(\rho^{-1} x-\sigma\right) \text {, } \\
& \rho=\frac{2^{3 / 2}\left(1+\left(1+a_{2}\right)^{2} a_{3}^{2}\right)^{1 / 2}}{\left(1+a_{2}\right)^{3 / 2}}, \sigma=\frac{a_{4}\left(1+a_{2}\right)^{3 / 2}\left(1+4 a_{2} a_{3}^{2}\right)}{4\left(1+\left(1+a_{2}\right)^{2} a_{3}^{2}\right)^{1 / 2}}, \\
& \sigma_{0}=2^{-1 / 2}\left(1+4 a_{2} a_{3}^{2}\right) a_{4}, \rho_{1}=\left(1+\left(1+a_{2}\right)^{2} a_{3}^{2}\right)^{1 / 2}, \\
& \sigma_{1}=\frac{a_{4}\left(1+4 a_{2} a_{3}^{2}-\left(1+\left(1+a_{2}\right)^{2} a_{3}^{2}\right)^{1 / 2}\right)}{2^{1 / 2}\left(1+\left(1+a_{2}\right)^{2} a_{3}^{2}\right)^{1 / 2}}, \rho_{2}=\frac{\left(1+\left(1+a_{2}\right)^{2} a_{3}^{2}\right)^{1 / 2}}{\left(1+a_{2}\right)^{3 / 2}\left(1+a_{3}^{2}\right)^{1 / 2}}, \\
& \sigma_{2}=\frac{a_{4}\left(1+a_{2}\right)^{3 / 2}\left(1+a_{3}^{2}\right)^{1 / 2}\left(1+4 a_{2} a_{3}^{2}\right)}{2^{1 / 2}\left(1+\left(1+a_{2}\right)^{2} a_{3}^{2}\right)^{1 / 2}}-2^{-1 / 2} a_{4}, \\
& \sigma_{3}=\frac{a_{4}\left(1+4 a_{2} a_{3}^{2}\right)}{2^{1 / 2}\left(1+\left(1+a_{2}\right)^{2} a_{3}^{2}\right)^{1 / 2}}, \sigma_{4}=\frac{a_{4}\left(1+a_{2}\right)^{3 / 2}\left(1+a_{3}^{2}\right)^{1 / 2}\left(1+4 a_{2} a_{3}^{2}\right)}{2^{1 / 2}\left(1+\left(1+a_{2}\right)^{2} a_{3}^{2}\right)^{1 / 2}}, \\
& \sigma_{5}=\frac{a_{4}\left(1+a_{2}\right)^{3 / 2}\left(1+4 a_{2} a_{3}^{2}\right)}{2^{1 / 2}\left(1+\left(1+a_{2}\right)^{2} a_{3}^{2}\right)^{1 / 2}}-2^{-1 / 2} a_{4} .
\end{aligned}
$$

The various parameter restrictions of the polynomial (8.1) are summarized in Fig. 7. Many boxes essentially coincide.

\subsection{From Wilson to Hermite along Continuous Dual Hahn}

Here we will see the chart for the Wilson manifold corresponding to the first graph in Fig. 3. The monic Wilson polynomial $w_{n}$ is given by (5.4). For $b_{1}, b_{2}, b_{3}, b_{4}>0$ put

$$
p_{n}(x)=p_{n}\left(x ; b_{1}, b_{2}, b_{3}, b_{4}\right):=\rho^{n} w_{n}\left(\rho^{-1} x-\sigma ; a, b, c, d\right),
$$

where

$$
\begin{aligned}
& a=\frac{1+b_{1}}{2 b_{1}}+\frac{1+4 b_{1} b_{2}}{2 b_{1}^{3} b_{2} b_{3}} i, \quad b=\frac{1+b_{1}}{2 b_{1}}-\frac{1+4 b_{1} b_{2}}{2 b_{1}^{3} b_{2} b_{3}} i, \\
& c=\frac{1}{b_{1}^{6} b_{2}^{2} b_{3}^{3} b_{4}}+\frac{2+b_{1} b_{3}+b_{1}^{3} b_{2} b_{3}^{2}+3 b_{1}^{4} b_{2} b_{3}^{2}}{2 b_{1}^{4} b_{2} b_{3}^{2}}
\end{aligned}
$$




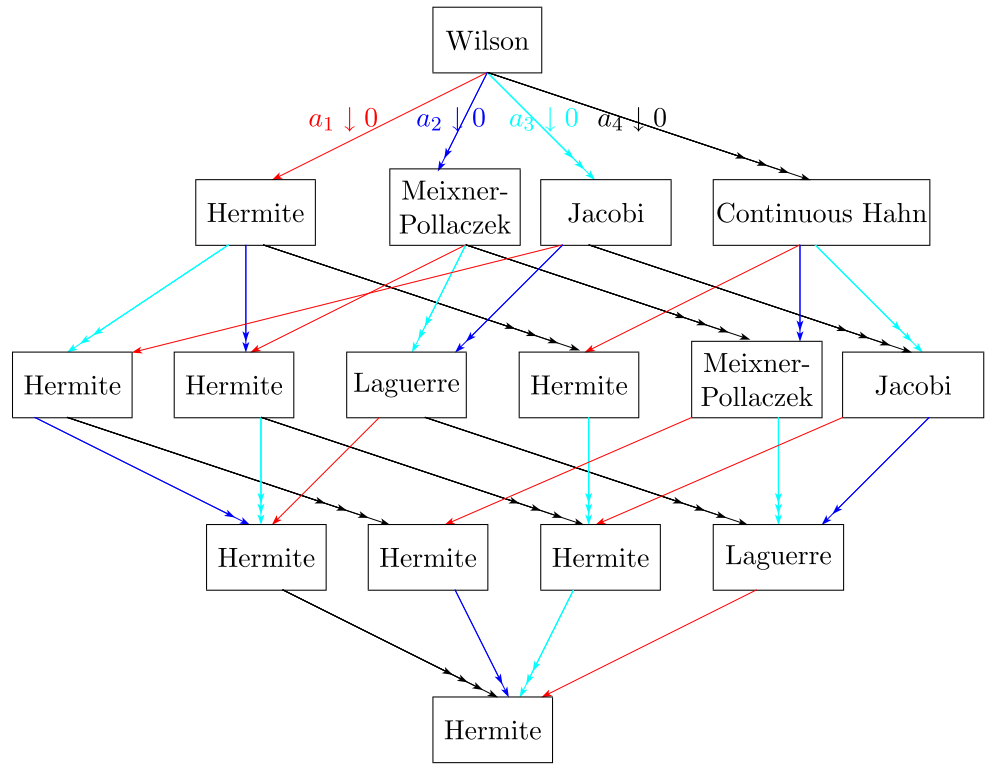

Fig. 7 The various parameter restrictions in the first Wilson manifold

$$
\begin{aligned}
& d=-\frac{2+b_{1} b_{3}+b_{1}^{3} b_{2} b_{3}^{2}+b_{1}^{4} b_{2} b_{3}^{2}}{2 b_{1}^{4} b_{2} b_{3}^{2}}, \\
& \rho=2^{-1 / 2} b_{1}^{9 / 2} b_{2} b_{3}^{2}, \\
& \sigma=-\frac{1+4 b_{1} b_{2}+4 b_{1}^{2} b_{2} b_{4}+4 b_{1}^{3} b_{2}^{2} b_{3} b_{4}+4 b_{1}^{4} b_{2}^{2} b_{3}^{2} b_{4}+4 b_{1}^{4} b_{2}^{2} b_{3} b_{4}^{2}+4 b_{1}^{5} b_{2}^{2} b_{3}^{2} b_{4}^{2}}{4 b_{1}^{6} b_{2}^{2} b_{3}^{2}} .
\end{aligned}
$$

Then (6.3) holds with

$$
\begin{aligned}
B_{n}= & 2^{-5 / 2} b_{1}^{1 / 2}\left(8 n^{4} b_{1}^{16} b_{2}^{5} b_{3}^{8} b_{4}^{2}+16 n^{3} b_{1}^{10} b_{2}^{3} b_{3}^{5} b_{4}\left(1+b_{1}^{5} b_{2}^{2} b_{3}^{3} b_{4}+b_{1}^{6} b_{2}^{2} b_{3}^{3} b_{4}\right)\right. \\
& +4 n^{2} b_{1}^{4} b_{2} b_{3}^{2}\left(2-2 b_{1}^{2} b_{2} b_{3} b_{4}-b_{1}^{3} b_{2} b_{3}^{2} b_{4}+5 b_{1}^{5} b_{2}^{2} b_{3}^{3} b_{4}+4 b_{1}^{6} b_{2}^{2} b_{3}^{3} b_{4}\right. \\
& -2 b_{1}^{4} b_{2}^{2} b_{3}^{2} b_{4}^{2}-2 b_{1}^{5} b_{2}^{2} b_{3}^{3} b_{4}^{2}-b_{1}^{6} b_{2}^{2} b_{3}^{4} b_{4}^{2}-2 b_{1}^{7} b_{2}^{3} b_{3}^{4} b_{4}^{2}-4 b_{1}^{8} b_{2}^{3} b_{3}^{4} b_{4}^{2} \\
& +8 b_{1}^{8} b_{2}^{4} b_{3}^{4} b_{4}^{2}-b_{1}^{8} b_{2}^{3} b_{3}^{5} b_{4}^{2}-2 b_{1}^{9} b_{2}^{3} b_{3}^{5} b_{4}^{2}+2 b_{1}^{10} b_{2}^{4} b_{3}^{6} b_{4}^{2}+4 b_{1}^{11} b_{2}^{4} b_{3}^{6} b_{4}^{2} \\
& +b_{1}^{12} b_{2}^{4} b_{3}^{6} b_{4}^{2}-4 b_{1}^{8} b_{2}^{3} b_{3}^{4} b_{4}^{3}-4 b_{1}^{9} b_{2}^{4} b_{3}^{5} b_{4}^{3}-4 b_{1}^{10} b_{2}^{4} b_{3}^{6} b_{4}^{3}-4 b_{1}^{10} b_{2}^{4} b_{3}^{5} b_{4}^{4} \\
& \left.-4 b_{1}^{11} b_{2}^{4} b_{3}^{6} b_{4}^{4}\right)-4 n\left(1+b_{1}^{5} b_{2}^{2} b_{3}^{3} b_{4}+b_{1}^{6} b_{2}^{2} b_{3}^{3} b_{4}\right)\left(2+b_{1} b_{3}-b_{1}^{3} b_{2} b_{3}^{2}\right. \\
& +2 b_{1}^{2} b_{2} b_{3} b_{4}+2 b_{1}^{3} b_{2} b_{3}^{2} b_{4}+b_{1}^{4} b_{2} b_{3}^{3} b_{4}+2 b_{1}^{5} b_{2}^{2} b_{3}^{3} b_{4}+4 b_{1}^{6} b_{2}^{2} b_{3}^{3} b_{4} \\
& -8 b_{1}^{6} b_{2}^{3} b_{3}^{3} b_{4}+b_{1}^{6} b_{2}^{2} b_{3}^{4} b_{4}+2 b_{1}^{7} b_{2}^{2} b_{3}^{4} b_{4}+b_{1}^{10} b_{2}^{3} b_{3}^{5} b_{4}+4 b_{1}^{6} b_{2}^{2} b_{3}^{3} b_{4}^{2} \\
& \left.+4 b_{1}^{7} b_{2}^{3} b_{3}^{4} b_{4}^{2}+4 b_{1}^{8} b_{2}^{3} b_{3}^{5} b_{4}^{2}+4 b_{1}^{8} b_{2}^{3} b_{3}^{4} b_{4}^{3}+4 b_{1}^{9} b_{2}^{3} b_{3}^{5} b_{4}^{3}\right) \\
& -\left(1+b_{1}^{5} b_{2}^{2} b_{3}^{3} b_{4}\right)\left(4-16 b_{2}+2 b_{3}+2 b_{1} b_{3}+b_{1}^{2} b_{2} b_{3}^{2}+2 b_{1}^{3} b_{2} b_{3}^{2}\right.
\end{aligned}
$$




$$
\begin{aligned}
& +b_{1}^{4} b_{2} b_{3}^{2}+4 b_{4}+8 b_{1} b_{2} b_{3} b_{4}+4 b_{1}^{2} b_{2} b_{3} b_{4}+8 b_{1}^{2} b_{2} b_{3}^{2} b_{4}+4 b_{1}^{3} b_{2} b_{3}^{2} b_{4} \\
& +2 b_{1}^{3} b_{2} b_{3}^{3} b_{4}+2 b_{1}^{4} b_{2} b_{3}^{3} b_{4}+8 b_{1}^{4} b_{2}^{2} b_{3}^{3} b_{4}+12 b_{1}^{5} b_{2}^{2} b_{3}^{3} b_{4}+8 b_{1}^{6} b_{2}^{2} b_{3}^{3} b_{4} \\
& -16 b_{1}^{6} b_{2}^{3} b_{3}^{3} b_{4}+2 b_{1}^{5} b_{2}^{2} b_{3}^{4} b_{4}+6 b_{1}^{6} b_{2}^{2} b_{3}^{4} b_{4}+4 b_{1}^{7} b_{2}^{2} b_{3}^{4} b_{4}+b_{1}^{7} b_{2}^{3} b_{3}^{5} b_{4} \\
& +4 b_{1}^{8} b_{2}^{3} b_{3}^{5} b_{4}+5 b_{1}^{9} b_{2}^{3} b_{3}^{5} b_{4}+2 b_{1}^{10} b_{2}^{3} b_{3}^{5} b_{4}+4 b_{1}^{2} b_{2} b_{3} b_{4}^{2}+4 b_{1}^{3} b_{2} b_{3}^{2} b_{4}^{2} \\
& +4 b_{1}^{5} b_{2}^{2} b_{3}^{3} b_{4}^{2}+8 b_{1}^{6} b_{2}^{2} b_{3}^{3} b_{4}^{2}+4 b_{1}^{6} b_{2}^{3} b_{3}^{4} b_{4}^{2}+8 b_{1}^{7} b_{2}^{3} b_{3}^{4} b_{4}^{2}+4 b_{1}^{7} b_{2}^{3} b_{3}^{5} b_{4}^{2} \\
& \left.\left.+8 b_{1}^{8} b_{2}^{3} b_{3}^{5} b_{4}^{2}+4 b_{1}^{7} b_{2}^{3} b_{3}^{4} b_{4}^{3}+8 b_{1}^{8} b_{2}^{3} b_{3}^{4} b_{4}^{3}+4 b_{1}^{8} b_{2}^{3} b_{3}^{5} b_{4}^{3}+8 b_{1}^{9} b_{2}^{3} b_{3}^{5} b_{4}^{3}\right)\right) \\
& \times\left(1+b_{1}^{5} b_{2}^{2} b_{3}^{3} b_{4}+2 n b_{1}^{6} b_{2}^{2} b_{3}^{3} b_{4}\right)^{-1} \\
& \times\left(1+b_{1}^{5} b_{2}^{2} b_{3}^{3} b_{4}+2(1+n) b_{1}^{6} b_{2}^{2} b_{3}^{3} b_{4}\right)^{-1}, \\
& C_{n}=\frac{1}{8} n\left(1+n b_{1}\right)\left(1+n b_{1}^{6} b_{2}^{2} b_{3}^{3} b_{4}\right)\left(1+b_{1}^{5} b_{2}^{2} b_{3}^{3} b_{4}\left(1+n b_{1}\right)\right) \\
& \times \frac{2+2 b_{1} b_{3}+b_{1}^{2} b_{3}^{2}+4 b_{1}^{3} b_{2} b_{3}^{2}+8 b_{1}^{4} b_{2}^{2} b_{3}^{2}+2(1-n) b_{1}^{4} b_{2} b_{3}^{2}\left(2+b_{1} b_{3}\right)+2(1-n)^{2} b_{1}^{8} b_{2}^{2} b_{3}^{4}}{\left(1+b_{1}^{5} b_{2}^{2} b_{3}^{3} b_{4}\left(1+(2 n-1) b_{1}\right)\right)\left(1+b_{1}^{5} b_{2}^{2} b_{3}^{3} b_{4}\left(1+2 n b_{1}\right)\right)^{2}\left(1+b_{1}^{5} b_{2}^{2} b_{3}^{3} b_{4}\left(1+(2 n+1) b_{1}\right)\right)} \\
& \times\left(2+4 b_{1}^{2} b_{2} b_{3} b_{4}+2 b_{1}^{3} b_{2} b_{3}^{2} b_{4}+4 b_{1}^{5} b_{2}^{2} b_{3}^{3} b_{4}+4(1+n) b_{1}^{6} b_{2}^{2} b_{3}^{3} b_{4}\right. \\
& +2 b_{1}^{4} b_{2}^{2} b_{3}^{2} b_{4}^{2}+2 b_{1}^{5} b_{2}^{2} b_{3}^{3} b_{4}^{2}+b_{1}^{6} b_{2}^{2} b_{3}^{4} b_{4}^{2}+8 b_{1}^{7} b_{2}^{3} b_{3}^{4} b_{4}^{2} \\
& +4(1+n) b_{1}^{8} b_{2}^{3} b_{3}^{4} b_{4}^{2}+8 b_{1}^{8} b_{2}^{4} b_{3}^{4} b_{4}^{2}+2 b_{1}^{8} b_{2}^{3} b_{3}^{5} b_{4}^{2}\left(1+(n+1) b_{1}\right) \\
& \left.+2 b_{1}^{10} b_{2}^{4} b_{3}^{6} b_{4}^{2}\left(1+(n+1) b_{1}\right)^{2}\right) \text {. }
\end{aligned}
$$

Note that $B_{n}$ and $C_{n}$, as functions of $b_{1}, b_{2}, b_{3}, b_{4}>0$, can be uniquely extended to continuous functions of $b_{1}, b_{2}, b_{3}, b_{4} \geq 0$.

We now set one or more of the $b_{1}, b_{2}, b_{3}, b_{4}$ equal to zero in (8.5) and (8.6) and we proceed as in Sect. 7.1. We obtain:

\section{Continuous dual Hahn,}

$$
\begin{aligned}
& p_{n}\left(x ; b_{1}, b_{2}, b_{3}, 0\right)=\rho^{n} s_{n}\left(\rho^{-1} x-\sigma ; a, b, c\right), \\
& a=\frac{1+b_{1}}{2 b_{1}}+\frac{1+4 b_{1} b_{2}}{2 b_{1}^{3} b_{2} b_{3}} i, b=\frac{1+b_{1}}{2 b_{1}}-\frac{1+4 b_{1} b_{2}}{2 b_{1}^{3} b_{2} b_{3}} i, \\
& c=-\frac{2+b_{1} b_{3}+b_{1}^{3} b_{2} b_{3}^{2}+b_{1}^{4} b_{2} b_{3}^{2}}{2 b_{1}^{4} b_{2} b_{3}^{2}}, \rho=2^{-1 / 2} b_{1}^{9 / 2} b_{2} b_{3}^{2}, \sigma=-\frac{1+4 b_{1} b_{2}}{4 b_{1}^{6} b_{2}^{2} b_{3}^{2}} ;
\end{aligned}
$$

\section{Meixner-Pollaczek,}

$$
\begin{aligned}
& p_{n}\left(x ; b_{1}, 0, b_{3}, b_{4}\right)=p_{n}\left(x-\sigma_{0} ; b_{1}, 0, b_{3}, 0\right)=\rho^{n} p_{n}^{(\lambda)}\left(\rho^{-1} x-\sigma\right), \\
& \lambda=\frac{1+b_{1}}{2 b_{1}}, \phi=\arctan \left(\frac{b_{1} b_{3}}{2+b_{1} b_{3}}\right), \rho=2^{-1 / 2} b_{1}^{3 / 2} b_{3}, \sigma=\frac{1-b_{1} b_{4}}{b_{1}^{2} b_{3}}, \\
& \sigma_{0}=-2^{-1 / 2} b_{1}^{1 / 2} b_{4}
\end{aligned}
$$




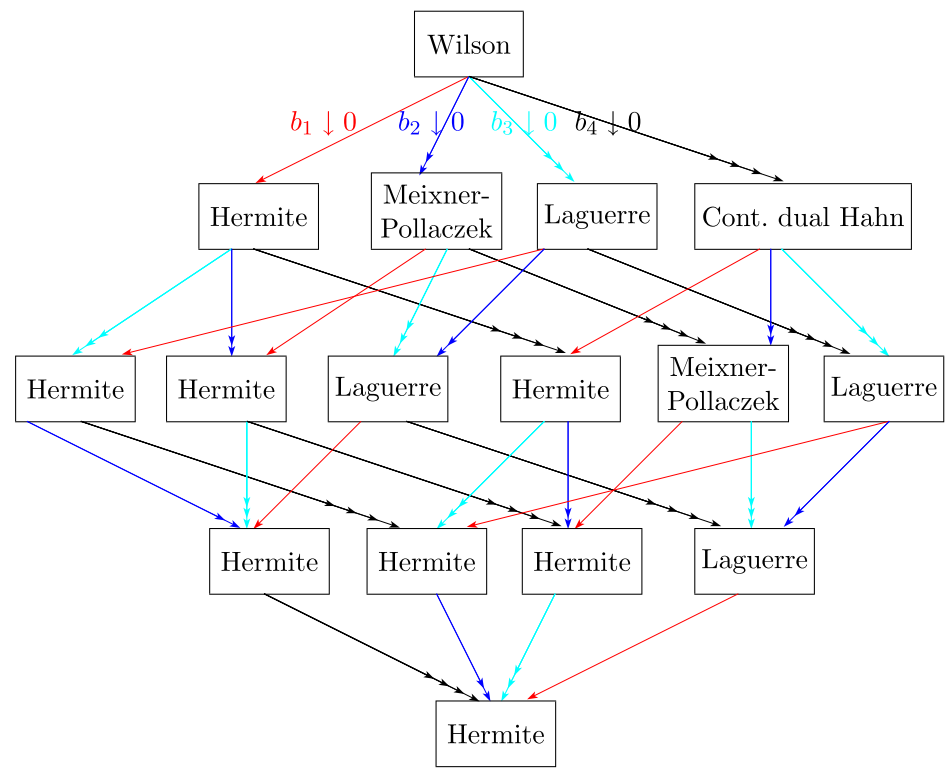

Fig. 8 The various parameter restrictions in the second Wilson manifold

\section{Laguerre,}

$$
\begin{gathered}
p_{n}\left(x ; b_{1}, b_{2}, 0, b_{4}\right)=p_{n}\left(x-\sigma_{0} ; b_{1}, 0,0, b_{4}\right)=p_{n}\left(x-\sigma_{1} ; b_{1}, b_{2}, 0,0\right) \\
=p_{n}\left(x-\sigma_{1} ; b_{1}, 0,0,0\right)=\rho^{n} \ell_{n}^{(\alpha)}\left(\rho^{-1} x-\sigma\right), \\
\alpha=b_{1}^{-1}, \rho=2^{-1 / 2} b_{1}^{1 / 2}, \sigma=b_{4}-4 b_{2}-b_{1}^{-1}, \sigma_{0}=2^{3 / 2} b_{1}^{1 / 2} b_{2}, \\
\sigma_{1}=-2^{-1 / 2} b_{1}^{1 / 2} b_{4}
\end{gathered}
$$

Hermite,

$$
\begin{aligned}
& p_{n}\left(x ; 0, b_{2}, b_{3}, b_{4}\right)=p_{n}(x ; 0,0,0,0)=\rho^{n} h_{n}\left(\rho^{-1} x-\sigma\right), \\
& \quad \rho=1, \sigma=0 .
\end{aligned}
$$

The various parameter restrictions of the polynomial (8.4) are summarized in Fig. 8. Many boxes almost coincide.

\section{Discussion of the results}

Various aspects of the results presented in this paper need further attention in the future. I list them below.

1. The various reparametrizations (three for the Racah polynomials and two for the Wilson polynomials) were obtained by computer algebra experiments: by varying 
possible reparametrizations until they "fit", i.e., until the coefficients $B_{n}$ and $C_{n}$ in the recurrence relation (6.3) are continuous on all parts of the boundary and give there $B_{n}$ and $C_{n}$ for families on lower levels of the Askey scheme. Is each of these reparametrizations canonical in some way, in the sense that it is unique, or generates all other reparametrizations which do the same job?

2. Do we really need three charts for covering the Racah manifold? In particular, does there exist a "fusion" of the second and third chart for the Racah manifold such that everything below dual Hahn, including Laguerre, is covered?

3. Gluing between the two Wilson manifolds should be considered on the threedimensional part corresponding with the three-parameter family of monic Wilson polynomials $w_{n}(x ; a, b, c, d)$ having one pair of complex conjugate parameters and the other two parameters equal and real. Also, gluing on lower dimensional parts between Wilson manifolds and the Racah manifold should be considered such that one or more of the boxes for Jacobi, Laguerre and Hermite in the various manifolds are identified.

4. As we have seen in Figs. 4-8, some families occur in various parts of the boundary in a given chart. Hence, our geometric description of the Askey scheme is not in bijective correspondence with the Askey scheme, but this will only be achieved by considering suitable quotient spaces of our four-manifolds with corners. A precise description of these quotient spaces should be worked out.

5. An obvious challenge would be to extend the results of this paper to the $q$-Askey scheme, including the limits for $q \uparrow 1$. A trial for a small part of the $q$-Askey scheme with its limits to $q=1$ would already be interesting.

Open Access This article is distributed under the terms of the Creative Commons Attribution Noncommercial License which permits any noncommercial use, distribution, and reproduction in any medium, provided the original author(s) and source are credited.

\section{References}

1. Askey, R., Wilson, J.A.: Some basic hypergeometric orthogonal polynomials that generalize Jacobi polynomials. Mem. Am. Math. Soc. 54, 319 (1985)

2. Askey, R.: Continuous Hahn polynomials. J. Phys. A 18, L1017-L1019 (1985)

3. Askey, R.: Limits of some $q$-Laguerre polynomials. J. Approx. Theory 46, 213-216 (1986)

4. Atakishiyev, N.M., Suslov, S.K.: The Hahn and Meixner polynomials of an imaginary argument and some of their applications. J. Phys. A 18, 1583-1596 (1985)

5. Cerf, J.: Topologie de certains espaces de plongements. Bull. Soc. Math. Fr. 89, 227-380 (1961)

6. Chihara, T.S.: An Introduction to Orthogonal Polynomials. Gordon and Breach, New York (1978)

7. Cooper, R.D., Hoare, M.R., Rahman, M.: Stochastic processes and special functions: on the probabilistic origin of some positive kernels associated with classical orthogonal polynomials. J. Math. Anal. Appl. 61, 262-291 (1977)

8. Dominici, D.: Asymptotic analysis of the Askey-scheme I: from Krawtchouk to Charlier. Cent. Eur. J. Math. 5, 280-304 (2007). arXiv:math/0501072v1 [math.CA]

9. Dominici, D.: Asymptotic analysis of the Askey-scheme II: from Charlier to Hermite (2005). arXiv:math/0508264v1 [math.CA]

10. Douady, A.: Variétés à bord anguleux et voisinages tubulaires. In: Séminaire Henri Cartan, 1961/62, Exposé 1

11. Ferreira, C., Lopez, J.L., Mainar, E.: Asymptotic relations in the Askey scheme for hypergeometric orthogonal polynomials. Adv. Appl. Math. 31, 61-85 (2003)

12. Ferreira, C., López, J.L., Pagola, P.J.: Asymptotic approximations between the Hahn-type polynomials and Hermite Laguerre and Charlier polynomials. Acta Appl. Math. 103, 235-252 (2008) 
13. Ferreira, C., López, J.L., Sinusía, E.P.: Asymptotic relations between the Hahn-type polynomials and Meixner-Pollaczek, Jacobi, Meixner and Krawtchouk polynomials. J. Comput. Appl. Math. 217, 88109 (2008)

14. Godoy, E., Ronveaux, A., Zarzo, A., Area, I.: On the limit relations between classical continuous and discrete orthogonal polynomials. J. Comput. Appl. Math. 91, 97-105 (1998)

15. Koekoek, R., Swarttouw, R.F.: The Askey-scheme of hypergeometric orthogonal polynomials and its $q$-analogue. Report 98-17, Faculty of Technical Mathematics and Informatics. Delft University of Technology (1998); http://aw.twi.tudelft.nl/ koekoek/askey/

16. Koepf, W., Schmersau, D.: Recurrence equations and their classical orthogonal polynomial solutions. Appl. Math. Comput. 128, 303-327 (2002)

17. Koornwinder, T.H.: Uniform multi-parameter limit transitions in the Askey tableau (1993). arXiv:math/9309213v1 [math.CA]

18. Labelle, J.: Tableau d'Askey. In: Brezinski, C., et al. (eds.) Polynômes orthogonaux et applications. Lecture Notes in Math., vol. 1171, pp. xxxvi-xxxvii. Springer, Berlin (1985)

19. López, J.L., Temme, N.M.: Approximation of orthogonal polynomials in terms of Hermite polynomials. Methods Appl. Anal. 6, 131-146 (1999)

20. Palamà, G.: Su delle relazioni integrali relative ai polinomi di Laguerre e d'Hermite. Rend. Semin. Mat. Univ. Padova 10, 46-54 (1939)

21. Ronveaux, A., Zarzo, A., Area, I., Godoy, E.: Transverse limits in the Askey tableau. J. Comput. Appl. Math. 99, 327-335 (1998)

22. Szegő, G.: Orthogonal Polynomials, 4th edn. Am. Math. Soc. Colloquium Publications, vol. 23. American Mathematical Society, Providence (1975)

23. Temme, N.M., López, J.L.: The Askey scheme for hypergeometric orthogonal polynomials viewed from asymptotic analysis. J. Comput. Appl. Math. 133, 623-633 (2001). arXiv:math/0109185v1 [math.CA]

24. Toscano, L.: Formule limiti sui polinomi di Laguerre. Boll. Unione Mat. Ital. 1, 337-339 (1939) 